\title{
Mechanical Properties in the Glioma Microenvironment: Emerging Insights and Theranostic Opportunities
}

\author{
Adip G. Bhargav ${ }^{1}$, Joseph S. Domino ${ }^{1}$, Roukoz Chamoun ${ }^{1}$ and Sufi M. Thomas ${ }^{2 *}$ \\ ${ }^{1}$ Department of Neurological Surgery, University of Kansas Medical Center, Kansas City, KS, United States, ${ }^{2}$ Department of \\ Otolaryngology, University of Kansas Medical Center, Kansas City, KS, United States
}

OPEN ACCESS

Edited by:

Bozena Kaminska,

Nencki Institute of Experimental

Biology (PAS), Poland

Reviewed by:

Serena Pellegatta,

IRCCS Carlo Besta Neurological Institute Foundation, Italy Ignacio Ochoa,

University of Zaragoza, Spain

*Correspondence:

Sufi M. Thomas

sthomas7@kumc.edu

Specialty section:

This article was submitted to Neuro-Oncology and

Neurosurgical Oncology,

a section of the journal

Frontiers in Oncology

Received: 30 October 2021 Accepted: 29 December 2021

Published: 21 January 2022

Citation:

Bhargav AG, Domino JS, Chamoun $R$ and Thomas SM (2022) Mechanical

Properties in the Glioma

Microenvironment: Emerging Insights and Theranostic Opportunities.

Front. Oncol. 11:805628.

doi: 10.3389/fonc.2021.805628
Gliomas represent the most common malignant primary brain tumors, and a high-grade subset of these tumors including glioblastoma are particularly refractory to current standard-of-care therapies including maximal surgical resection and chemoradiation. The prognosis of patients with these tumors continues to be poor with existing treatments and understanding treatment failure is required. The dynamic interplay between the tumor and its microenvironment has been increasingly recognized as a key mechanism by which cellular adaptation, tumor heterogeneity, and treatment resistance develops. Beyond ongoing lines of investigation into the peritumoral cellular milieu and microenvironmental architecture, recent studies have identified the growing role of mechanical properties of the microenvironment. Elucidating the impact of these biophysical factors on disease heterogeneity is crucial for designing durable therapies and may offer novel approaches for intervention and disease monitoring. Specifically, pharmacologic targeting of mechanical signal transduction substrates such as specific ion channels that have been implicated in glioma progression or the development of agents that alter the mechanical properties of the microenvironment to halt disease progression have the potential to be promising treatment strategies based on early studies. Similarly, the development of technology to measure mechanical properties of the microenvironment in vitro and in vivo and simulate these properties in bioengineered models may facilitate the use of mechanical properties as diagnostic or prognostic biomarkers that can guide treatment. Here, we review current perspectives on the influence of mechanical properties in glioma with a focus on biophysical features of tumor-adjacent tissue, the role of fluid mechanics, and mechanisms of mechanical signal transduction. We highlight the implications of recent discoveries for novel diagnostics, therapeutic targets, and accurate preclinical modeling of glioma.

Keywords: glioma, heterogeneity, tumor microenvironment, biophysical properties, tissue mechanics 


\section{INTRODUCTION}

\section{Contemporary Management of Malignant Glioma and Biological Considerations}

Mortality due to cancer continues to rise worldwide with improving medical management of other disease processes. Brain cancer, specifically, represents one of the most lethal cancer subtypes. Malignant gliomas are a group of primary brain tumors that harbor a poor prognosis for afflicted patients $(1,2)$. Though there is some variation in survival rates ranging from months to decades among the different histological and molecular categories and grades of gliomas, in general, current therapies are not curative. Within this group is a subset of particularly high-grade tumors including glioblastoma which portend the worst survival with recent estimates of median survival at 8 to 14 months and a 7.2\% 5-year survival rate post-diagnosis $(1,3)$. Unfortunately, this group also comprises the most common type of malignant glioma accounting for approximately $48.6 \%$ of all primary malignant brain tumors (1).

The standard of care and outcomes for glioblastoma have been largely unchanged since the development of the Stupp protocol (4, 5). Contemporary management of glioblastoma aims for maximal cytoreductive surgery while preserving critical neurologic function that is followed by adjunctive chemotherapy with temozolomide and fractionated radiotherapy (6). In certain cases, this treatment algorithm is limited by patient and disease factors including fitness to undergo aggressive therapy and tumor location, respectively. In such cases, intervention is directed towards establishing a definitive diagnosis as with biopsy and mitigating symptoms (6).

In light of poor outcomes in patients with glioblastoma, several lines of investigation are ongoing in order to develop novel therapeutics and treatment strategies (7-12). Importantly, advances in the understanding of tumor biology and influences of the microenvironment have begun to inform emerging paradigms for management of glioma. Glioma stem cells or brain tumor-initiating cells (BTICs) have been established as a subset of cells within glioma that contribute to treatment resistance and recurrence of disease. These cells exhibit properties including chemoresistance and radioresistance as well as considerable heterogeneity and plasticity on multiple levels which has posed a therapeutic challenge (13-18). Heterogeneity of BTICs encompasses variation in tumor characteristics over time-temporal heterogeneity, variation in tumor and cellular characteristics depending on location within a tumor-locoregional heterogeneity, and variation in disease characteristics from patient to patient-population heterogeneity which can impact response to treatment. As a result, current investigations are transitioning from single-agent or single-target therapies to treatment modalities with robust mechanisms of action that may overcome disease heterogeneity. Similarly, robust mechanisms of action are required to bypass tumor plasticity and changes in response to unimodal therapies $(8,17$, 19-21). Examples of such efforts include cell-based therapies, immunotherapy approaches, and bioengineering strategies such as gene therapy, and excellent overviews of these topics can be found elsewhere $(8,9,11,22-24)$.
Improved understanding of plasticity and heterogeneity of BTICs has also led to further study of the tumor microenvironment and its inherent heterogeneity and plasticity. Similar to BTICs, studies have demonstrated that cellular and vascular components of the microenvironment respond to tumorigenesis and treatment in ways that may be facilitating malignant adaptation in tumor cells (16, 25-27). The potential bidirectional interaction between tumor cells and tumor microenvironment is particularly evident when considering the influence of immune-active cells such as myeloid-derived suppressor cells on immunosuppression via immune cell dysregulation (16, 17, 25, 28). Although cellular and vascular niches in the tumor microenvironment are now known to be a key player in the tumor milieu, the study of the mechanical properties of the microenvironment is relatively new. As with other components of the microenvironment, evidence suggests an important evolving role for mechanical properties in the context of treatment resistance and disease.

\section{Tissue Mechanics in the Brain}

Tissue mechanics broadly comprise cellular and tissue stiffness properties as well as stresses transmitted by fluid including cerebrospinal fluid (CSF) dynamics and interstitial fluid pressures. The influence of tissue mechanics on normal brain development and homeostasis has been well-described $(29,30)$. In the developing brain, stiffness gradients arise during various stages of embryogenesis and migration of neural precursors and neural stem cell populations particularly in the subventricular zones of the brain. Gradients have been attributed to maturation of cellular cytoarchitecture and changes in extracellular matrix (ECM) composition that facilitate normal migratory and tissue organization in development (31-34). As a result, a general trend towards increased global brain stiffness is seen with notable regions of 'softer' brain such as the hippocampus in the adult brain (35-38). In addition to spatial organization of cells and tissue, heterogeneity of tissue mechanics in non-disease states is important for directing differentiation and cell-type determination of embryonic neural stem cells as well as adult stem cells. Notably, aberrant mechanical signaling from the microenvironment has tremendous implications for regulating the behavior and plasticity of BTICs and preclinical models of BTICs that is discussed in detail in subsequent sections $(29,30)$.

\section{Tissue Mechanics in Disease and Cancer}

Alteration of the inherent spatiotemporal heterogeneity during development and maturation contributes to various disease states including traumatic brain injury, neurodegenerative diseases, and cancer $(29,39,40)$. Briefly, studies demonstrate a progressive loss of global brain stiffness in the context of neurodegenerative disease that is contrary to generalized stiffening of the brain in aging, and this is secondary to cellular injury and compromise of cell-intrinsic mechanical factors as well as cell-extrinsic factors such as breakdown of the basement membrane in certain disease processes and changes in the composition of the ECM $(29,41-44)$. Conversely, preliminary studies demonstrate elevation in pro-stiffening factors such as 
Tenascin-C (TNC) in the setting of traumatic brain injury with evidence suggesting enhancement of mechanical signaling likely owing to changes in intracranial pressure, injury from intracranial hemorrhage, and direct injury to areas of adult neurogenesis that may affect long-term outcomes and neurodegeneration $(29,39,40)$.

The study of physical traits and microenvironmental mechanics in cancer is a relatively young field compared to research surrounding the traditional hallmarks of cancer. In recent years, the importance of physical characteristics in addition to biological factors has been increasingly recognized and extensively investigated in several cancers including breast cancer and carcinomas of the gastrointestinal system. From this work, ECM stiffness has emerged as a physical hallmark of many cancers that contributes to tumorigenesis, metastasis, metabolism, immune response and numerous additional processes (45-50). Though discussion of tissue mechanics in other cancer types is beyond the scope of this review, we highlight important principles gleaned from work in other cancers that may offer a template for further investigation of tissue mechanics in glioma, which is comparatively in its early stages. Comprehensive reviews of these fundamental discoveries and the work leading to these findings can be found elsewhere $(46,47,51,52)$. Investigation of tissue mechanics of the cancer microenvironment in various models has illuminated three biophysical concepts: 1) solid stress 2) fluid stress and 3) stiffness. Solid stress refers to amount of force per area present in the region of interest whether it is the tumor or the surrounding milieu $(52,51)$. The key factor influencing solid stress is derived from tumor tissue and cellular properties, though there is also contribution from ECM and surrounding components. Increased proliferation of cells within a tumor transmits increased stress through the spacelimited tumor microenvironment. In addition, spatial and geometric considerations can also exacerbate regional solid stress based on the alignment of cellular cytoskeletal components relative to ECM matrix components as well as through a mechanism referred to as "jamming" whereby cumulative stress is increased after a critical cell population is reached that augments cell to cell contact and force $(46,51,52)$. Fluid stress is the result of perturbation of interstitial fluid pressure as well as shear flow in certain microenvironments such as adjacent to the ventricles of the brain. Leaky tumor vasculature, impaired lymphatic drainage, and vascular compression secondary to solid stress can all contribute to increased interstitial pressure $(51,52)$.Global elevation in fluid stress as in the case of increased intracranial pressure in the fixed volume of the cranium can also exacerbate fluid stress at the tissue level. Finally, stiffness refers to the resistance to deformation as a result of stress and can be used to describe the tumor as a whole, individual cells, or the microenvironment and its components including the ECM. Global tissue stiffness is affected by ECM deposition or degradation, ECM cross-linking and changes in microarchitecture, and at the cellular level by cytoskeletal remodeling and cell contraction $(45,51,52)$.

Interdependence between certain hallmarks of cancer and physical traits in the microenvironment is a relatively unexplored area in brain cancer but has been described in other cancer types $(47-49,52)$. Recent studies have revealed a potential link between immune escape and tissue mechanics where regional stress may impair vascular and lymphatic channels $(49,51,52)$. This results in reduced migration of immune effector cells to the tumor site and effectively creates a functional immune escape phenomenon. Relatedly, cellular deformation secondary to solid and fluid stress may affect the integrity of intracellular structures including the nucleus and alter expression of immune soluble factors through direct physical perturbation as well as through mechanisms of mechanotransduction that affect downstream genetic and epigenetic regulation $(49,51,52)$. A similar type of interdependence is observed with tumor metabolism and tissue mechanics; it is possible that solid and fluid stress may significantly predispose tumor cells towards aberrant metabolism in a feed-forward mechanism that continues to progress as the tumor grows and microenvironmental stress increases. One example of this is the Warburg effect and the interplay between stress and aerobic glycolysis: increased regional stress may promote hypoxia via vascular compression within the tumor and thereby apply selection towards aerobic glycolysis to facilitate tumor growth and progression (48). Overarching these overlapping mechanisms is the concept of mechanoreciprocity which mirrors the dynamic seen between cancer cells and the biological factors of the microenvironment such as the dynamic interactions between BTICs and immune cells or BTICs and neurons in the tumor milieu $(17,46)$. In the same way, the interaction between cancer cell and physical properties of the microenvironment is also dynamic and has been most extensively documented in the process of cancer migration where reciprocal signal transduction and physical changes at the cellular level and tissue level enable the requisite cellular geometric changes, elasticity, and focal adhesions to achieve metastasis $(47,51,52)$. This dynamic interaction acts as the substrate for heterogeneity and plasticity in the physical traits of the tumor and microenvironment much like biological receptor- or soluble factor-mediated cell communication and therapy-induced changes to the cellular phenotype or genetics.

\section{BIOPHYSICAL FEATURES OF GLIOMA-ADJACENT TISSUE}

\section{Current Understanding of Tissue Mechanics in the Glioma Microenvironment}

Tissue mechanics in the glioma microenvironment primarily refers to solid stress from contributions by the surrounding ECM and tissue architecture as well as the cellular compartment in the tumor milieu which includes glioma cells. Unlike many other systemic cancers where the causative factors of solid stress may be more intuitive owing to typical growth patterns characterized by displacement of surrounding tissue via mass effect, gliomas tend to exhibit an infiltrative growth pattern. The components of physical stress generation in the microenvironment were unknown until recently. These are summarized in Figure 1. 


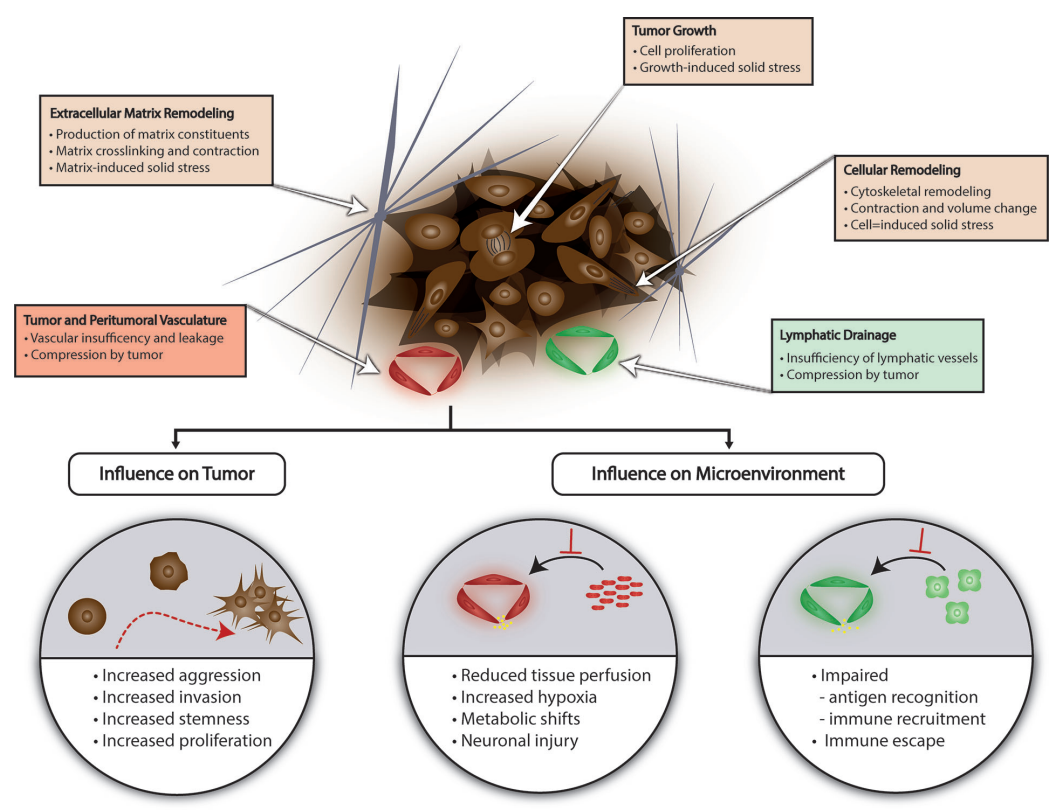

FIGURE 1 | Tissue Mechanics in Glioma. Several factors contribute to generation of solid stress in the glioma microenvironment including lymphatic or vascular insufficiency, tumor growth, and ECM or cellular remodeling. Solid stress can promote glioma aggression and create a microenvironment conducive for immune escape and BTIC selection. ECM, extracellular matrix; BTIC, brain tumor-initiating cell.

Stylianopoulos et al. developed a mathematical model to calculate growth-induced solid stress in a tumor by measuring the extent of deformation of tumor as stress is released from the tumor microenvironment after a cut is made along the long axis of the tumor (53). Various orthotopic cancer lines were employed to assess tumor-related solid stress and notably the U87 human glioblastoma cell line. Briefly, inoculation of tumor was performed in the flank of immunocompromised mice, and tumor was excised after reaching a tumor size of $1 \mathrm{~cm}^{3}$. The solid stress-release assay was performed in different iterations after treatment with agents to selectively deplete individual components of the tumor microenvironment and thereby identify the contributory factors to solid stress. Selective depletion of U87 cancer cells or collagen in the ECM produced almost a two-fold decrease in tumor opening, a surrogate measurement for solid stress compared to control tumor. Importantly, no relationship between interstitial fluid pressure and solid stress was noted suggesting that cellular and ECM factors are the primary contributors (53). Nia et al. later developed an alternate method to characterize growth-induced solid stress in situ as well as ex vivo measurement (54). For ex vivo measurement, following tumor excision in the manner aforementioned, tumor was embedded in agarose gel. A planar cut is then performed, and the 2-dimensional planar deformation of the tumor is measured using an ultrasound probe. In situ assessment of solid stress in a murine orthotopic model of U87 GBM was subsequently performed by using a cylindrical punch to excavate a component of the tumor through the cranial window and measuring deformation with ultrasound.
Interestingly, initial ex vivo 2-dimensional mapping of embedded U87 GBM demonstrated greater compressive stress at the periphery compared to the central core. Additionally, these tumors experienced less solid stress compared to other tumor types (maximum stress of $0.21 \mathrm{kPa}$ versus $7 \mathrm{kPa}$ in pancreatic tumors) (54). In situ measurement of solid stress revealed a significant influence of the surrounding tumor microenvironment-the cranial vault with fixed volume and surrounding normal tissue. Lesser degree of deformation was observed ex vivo compared to in situ measurement and approximately $0.02 \mathrm{kPa}$ and $0.1 \mathrm{kPa}$ compressive forces, respectively (54). This finding confirms the importance of considering the cranial vault as a fixed volume and accounting for the consequences when examining tissue mechanics in brain tumors. For example, states with increased intracranial pressure due to ancillary causes such as obstructive hydrocephalus may impact glioma microenvironment solid stress. Stylianopoulos et al. utilizing the previously described tumor relaxation model and mathematical model that the amount of deformation in U87 GBM tumors is proportional to the stored solid stress in the tumor microenvironment, and that solid stress forces in the periphery contribute to vascular and lymphatic collapse (55). This represents a possible mechanism based in the mechanical properties of the tumor microenvironment for BTIC selection and plasticity; impaired perfusion and lymphatic drainage may promote hypoxia within the tumor and a milieu conducive for aggressive, resistant cellular phenotypes as well as secondarily creating a physical barrier for potential cellular therapy or conventional chemotherapy (Figure 1). Seano and Nia et al. 
relate tumor growth pattern to the amount of solid stress imposed on surrounding normal brain tissue using in situ, ultrasound-based measurement of stress (56). They utilize two distinct GBM cell lines U87 and MGG8 which exhibit nodular and infiltrative growth, respectively in an orthotopic murine model. Analysis of solid stress in these groups revealed qualitative deformation of surrounding tissue on histology with U87 nodular tumors, but not MGG8 tumors. This was corroborated with stress measurements that showed lesser radial and circumferential stress in the MGG8 infiltrative tumors compared to U87 nodular tumors (MGG8: radial stress $0.014+/-0.001 \mathrm{kPa}$, circumferential stress $0.063+/-0.004 \mathrm{kPa}$; U87: radial stress $0.020+/-0.001 \mathrm{kPa}$, circumferential stress $0.110+/-0.005 \mathrm{kPa})(56)$.

Although the fundamental mechanisms of growth-induced solid stress and the variables affecting this has been elucidated over the past decade, investigating these features in humans within a complex biophysical system continues to be a challenge. In large part due to limitations of current technologies, noninvasive methods of assessing physical properties of intracranial tumors are still being optimized by several groups (57-61). Initial studies have begun to define the biophysical properties of glioma, albeit with some discrepancy among groups. Specifically, the stiffness of glioma tissue and the microenvironment is debated, contrary to other systemic cancers where tumor stiffness is a hallmark. Schregel et al. characterized physical parameters of orthotopic G30 BTIC cell line using magnetic resonance elastography (MRE) (62). Analysis of MRE parameters revealed significant heterogeneity within tumor tissue as indicated by viscoelastic modulus and shear wave speed; stiffer areas on MRE corresponded to regions of high cell density on histology, and softer areas corresponded to regions necrosis and lower cell density (62). In vivo, intraoperative MRE of brain tumors performed by some groups suggested increased stiffness of brain tumor compared to normal brain as well as a trend of increasing stiffness with lower grade tumors $(57,58)$. Notably, these were the only studies to utilize MRE intraoperatively. Chauvet et al. report young's modulus ranging from $11.4 \mathrm{kPa}$ to $33.1 \mathrm{kPa}$ by shear wave elastography including meningiomas, low-grade gliomas, highgrade gliomas, and metastases. Meningiomas exhibited the greatest stiffness $(33.1+/-5.9 \mathrm{kPa})$ whereas high-grade gliomas exhibited the least stiffness $(11.4+/-4.9 \mathrm{kPa})$; however, tumors on average were stiffer than normal brain stiffness of $7.3+/-3.6$ $\mathrm{kPa}$ (58). Other groups report similar trends with tumor grade, but report glioma tissue as softer than normal brain tissue though there is a small subset of gliomas that are stiffer in these studies (59-61). Reiss-Zimmerman et al. observe no significant difference in the elasticity parameter with MRE among tumor types whereas the elasticity component reflects the trend reported in the other studies-meningiomas exhibiting the least elasticity likely owing to higher cellular density compared to infiltrative gliomas. In this study, average young's modulus for all tumors was $1.43+/-0.33 \mathrm{kPa}$ while the average for normal white matter was 1.62 +/- 0.27 (59). Moreover, intertumoral heterogeneity and intratumoral heterogeneity in viscoelastic properties were evident among gliomas as well as meningiomas to a lesser degree and may be due to high rates of cell turnover and heterogeneous distribution of cell density in gliomas (59).

The biological substrate of tissue stiffness also requires special consideration in the context of gliomas. Typical constituents of the ECM found elsewhere in the body are absent in the brain, namely collagen, fibronectin, and laminin (63-65). Instead, proteoglycans as well as heparin sulfate and hyaluronic acid are present. Proteins involved in cellular adhesion, particularly TNC, are also present and these constituents are frequently enriched in gliomas which can contribute to ECM stiffness (63-65). The orchestration of changes in glioma microenvironment stiffness and the subsequent mechanotransduction remains unclear. Although aberrant production of ECM constituents, even in the absence of typical proteins such as collagen in the brain, likely plays a role in promoting glioma tissue stiffness, other mechanisms have also been described. Pogoda et al. report a phenomenon of compression stiffening in glioma tissue obtained from biopsy specimens of patients with GBM (66). They demonstrate that the young's moduli measured in patient GBM tissue is not significantly different from the viscoelasticity of normal mouse brain tissue which was used as a proxy for normal human brain. With the addition of compressive force, the relative young's modulus for GBM tissue compared to normal brain tissue increased significantly with increasing compressive force to a relative young's modulus of approximately 1.8 at $20 \%$ compression of tissue (66). Additionally, using the LN229 GBM cell line, Pogoda et al. showed cell stiffness and morphology was dependent on substrate stiffness which was assessed by gel cultures of varying stiffness (66). Compared to normal astrocytes, a monotonic dependence of cell adherent area on substrate shear modulus ranging from 300 to $14,000 \mathrm{~Pa}$ was observed. A similar relationship was observed with cell stiffness with a maximal cell stiffness of approximately $5 \mathrm{kPa}$ achieved at $14 \mathrm{kPa}$ of substrate stiffness after which no further changes in cellular stiffness was observed (66). Taken together, these findings suggests that although glioma tissue may be softer at baseline compared to surrounding tissue, regional normal brain stiffness can stimulate local changes in glioma stiffness signifying cytoskeletal remodeling and motility. A compression-driven mechanism of focal glioma stiffness may complement previously recognized mechanisms of primary ECM stiffening due to changes in ECM composition and deposition which is likely not the sole mediator of glioma stiffness. Compression from surrounding normal brain tissue can arise from various processes including increased intracranial pressure or interstitial pressures which are not uncommon in intracranial malignancy.

A recent MRE-based study and mathematical analysis by Streitberger et al. offer further reconciliation of relative glioma "softness" compared to normal brain and tumor progression and invasion (67). The authors first devised a phantom model mimicking the ECM and cytoarchitecture of meningioma and glioma using amalgamations of agar, heparin, and tofu. Viscoelastic properties were measured using MRE at varying 
solid composition ratios of the model and varying water content to determine how the viscoelastic properties of an intracranial tumor may change in response to increased fluid due to disrupted blood-brain barrier in surrounding vasculature (67). In their phantom models, different substrates functionally recapitulated different components of glioma ECM-abundant glucosaminoglycans (GAGs), absence of significant fibrosis in the case of heparin while the tofu phantom mimicked an entanglement of proteins governed by hydrophobic interactions with the high collagen content observed in meningioma with relatively high viscosity and increased fluidity (67). With glioma and the heparin phantom model, an inverse relationship between water absorption and viscosity was observed (67). Indeed, this was corroborated with MRE imaging studies in patients where T2 signal reflected higher water content and this corresponded to lower viscosity, i.e. lower phase angle and consequently decreased fluidity. Interestingly, other groups have also shown that increased ADC signal in GBM corresponding to higher water content may be associated with worse outcomes $(68,69)$. Based on these findings, the authors posit that GBM behaves functionally as a low viscosity, low fluidity solid secondary to the ability of significant, hydrophilic GAGs in the ECM to bind to water without meaningful perturbation of viscosity (67). On the other hand, meningioma behaves like a high viscosity, high fluidity fluid with ECM consisting of entangled proteins with hydrophobic aggregation. In the case of meningioma, the variation in focal shear stress can steeply increase stiffness due to local drainage of water followed by direct solid-solid contact of ECM constituents and protein coagulation (67). With regards to glioma infiltrative growth and tumor progression, viscous fingering. Briefly, in viscous fingering, the less viscous glioma with lower regional surface tension is pushed into the higher viscosity surrounding producing the characteristic microinvasive infiltration without clear margins (67). Overall, these studies suggest a unifying theory though additional work is required to elucidate the impact of tumor subtype, tumor location such as proximity to the ventricle, tumor/microenvironment heterogeneity, among other factors.

\section{Influence of Microenvironmental Tissue Mechanics on Glioma Malignancy}

Though studies examining the impact of tissue mechanics are limited, this is an area of considerable interest and active research with themes beginning to emerge. Unsurprisingly, given discoveries in tissue mechanics in other types of cancers, tissue mechanics in glioma tend to regulate hallmarks of cancer. Here we highlight representative studies that have uncovered these mechanisms (Figure 1).

Several studies have linked alterations in microenvironmental rigidity to enhanced glioma aggression, and some have also identified certain microenvironmental features as prognostic markers for survival at the patient level (70-73). Miroshnikova et al. established the importance of HIF $1 \alpha$ signaling, IDH status, and ECM components in GBM (70). Using in vitro models as well as human tumor samples, the authors show that increasing in
ECM stiffness is observed with increasing grade of tumor in glioma ranging from $50-1,400 \mathrm{~Pa}$ in low grade gliomas to 70 13,500 in higher grade gliomas; analysis of human samples revealed worst patient prognosis with tumors with a high proportion of stiff ECM $>1,400 \mathrm{~Pa}$ (70). Mechanistically, they define TNC and hyaluronic acid (HA) as key contributors to ECM stiffness in glioma and show increased levels of these constituents is associated with worse survival and stiffer tumor ECM. Finally, they describe the downstream effects of HIF1 $\alpha$ expression in a hypoxic tumor microenvironment (70). HIF1 $\alpha$ serves as a regulator of TNC expression and ultimately glioma microenvironment mechanical properties through stiffening of the ECM. At the tumor level, the authors show that perturbation of TNC-mediated ECM stiffening or at a point in the regulatory pathway with HIF $1 \alpha$ or one of its regulators miR-203 improved survival is observed in murine xenograft models of GBM. Notably, wild-type IDH via onco-metabolite (R)-2-hydroxyglutarate is implicated in HIF1 $\alpha$ regulation and consequently ECM stiffness regulation; in patients with recurrent IDH-mutant tumor, ECM stiffness was noted to increase comparing initial diagnosis and associated increased TNC expression suggesting a mechanosignaling-induced tumor aggression (70). Chen et al. similarly define a key mechanosignaling axis involving the PIEZO1 ion channel that has been shown to be overexpressed in a variety of cancers including all subtypes and grades of glioma signifying a potential common, evolutionarily conserved mechanosensation mechanism in cancer (71). Beginning with a Drosophila model of glioma and subsequently utilizing GBM and BTIC cell lines, the authors demonstrated that the PIEZO1 channel is necessary for tumor growth in vitro and in vivo and abrogation of the ion channel resulted in significantly longer survival and reduced tumor growth in mouse models (71). Further characterization of PIEZO1 as a central hub in a mechanotransduction cascade revealed subcellular location of the channel at sites of focal adhesion and a feed-forward mechanism whereby PIEZO1 was shown to be essential for ECM stiffening via regulation of other ECM remodeling genes including TAZ and FHL3 as well as glioma cell mechanotransduction (71). In experiments with PIEZO1 knockdown, stiffness-dependent glioma cell growth was not observed, and in experiments assessing the stiffness-dependent growth of glioma cells in response to varying stiffness hydrogels, increased expression of PIEZO1 was observed (71). Other groups have also confirmed the influence of microenvironmental stiffness on glioma growth and proliferation in the context of other signaling pathways including the EGFR and Rho/GTPase signaling pathways (72-74).

In addition to promoting glioma growth and proliferation, mechanical cues from the microenvironment also enhance other aspects of glioma stemness, specifically migration and invasion which enables diffuse spread through brain parenchyma (75-77). Zhang et al. elucidate a mechanism involving the cytokine IL-33 and receptor ST2 in the tumor milieu that stimulates expression and accumulation of TNC via NF- $\mathrm{KB}$ signaling in the microenvironment (76). Using a transwell migration assay, they demonstrate that IL-33 treatment produces a nearly 3 -fold 
increase in invaded cells in a TNC-dependent matter; the authors observed enhanced migration on in vivo histologic analysis as well (76). Kim et al. identify another mechanosensation mediator in the CD44-HA interaction that also enhances glioma cell invasion (77). Aberrant and increased expression of CD44 and $\mathrm{HA}$ is present in the glioma microenvironment, and Kim et al. show that this ligand-mediated interaction is distinct from other adhesion interactions as with integrins and is stiffness-dependent (77). Using U373-MG glioma cells in transwell invasion assay and time-lapse microscopy, the authors show that the migration speed and invasion properties of these cells in the presence of CD44/HA improves with increasing stiffness of the HA hydrogel in which they are cultured (77). Integrin-mediated interaction between glioma cells and microenvironment components such as the glycocalyx or BCL9L have also been described and shown to enhance glioma stemness $(75,78,79)$. Notably, in these studies, glioma stemness also incorporates the treatment-resistant quality of this phenotype and the authors show treatment sensitization employing gain- or loss-of-function methodologies to disturb the specific integrin-mediated mechanosensation (78, 79). Barnes et al. specifically examine recurrent GBM and determine that a bulky glycocalyx in the tumor microenvironment, that is frequently seen in recurrent GBM, interacts with integrins with mechanoreciprocity whereby downstream signaling enhances stemness of the cell which in turn effects increased tension in the glycocalyx to form a feedback loop (79).

Interestingly, in investigations by Miroshnikova et al. and others, GBM tumor tissue was observed to be stiffer than normal tissue and a trend was observed towards increased stiffness with high grade; in contrast, previously discussed studies found gliomas to be softer compared to normal brain and to exhibit the opposite trend with grade $(59,60,66,67,70,78)$. This underscores the complexity of measuring mechanical properties of the glioma microenvironments and requires further study to elucidate with consideration of standardized measurement techniques and preclinical modeling. Moreover, this may also be an indicator of the challenge associated with defining features of a heterogeneous disease process including sampling bias of the tumor as well as other confounders such as patient comorbidities and history of treatment.

Microenvironmental tissue properties also induce changes in tumor-adjacent tissue compartments in addition to glioma cells (Figure 1). Seano and Nia et al. show that glioma tumor growth in an orthotopic murine model compresses tumor-adjacent vasculature and decreases perfusion (56). They note that this effect is more pronounced in glioma cell lines characteristic for nodular growth pattern-U87, GL261, and BT474 compared with cell lines that exhibit infiltrative growth. For example, at a 20-day timepoint, the authors demonstrate that the nodular cell line U87 resulted in a significantly reduced intravital perfused blood volume fraction compared to baseline $(0.35$ to 0.2$)$ in surrounding vasculature whereas no significant changes were observed in the infiltrative cell line MGG8 in the same time frame (56). In tumors models with nodular growth, perfusion in the surrounding vasculature is also inversely correlated with tumor growth (56). MRI-based analysis of perfusion in tumor adjacent regions, not including peritumoral T2/FLAIR signal in a cohort of patients with GBM confirmed reduced perfusion in up to $53 \%$ of patients (56). Histological and behavioral analysis after tumor-related brain compression in the intracranial tumor mouse models also revealed evidence of neuronal injury and neuroinflammation as well as concordant significant changes in locomotion and gait suggesting deleterious neuronal sequelae from the physical effects of tumor growth (56). Tumor-related mechanical changes in the microenvironment can also promote local immune dysfunction. This type of microenvironment-mediated immunosuppression has been characterized in a variety of cancers and may result from tumor-related solid stress transmitted through the microenvironment as well as soluble factors and interactions with tumor-associated vasculature (80). In glioma specifically, Huang et al. show GBM ECM-based inhibition of $\mathrm{T}$ cell migration into the tumor milieu. Furthermore, they define an inverse correlation between TNC expression in the ECM and T cell transmigration (81). Increased levels of TNC in vivo in a mouse model of GBM was associated with reduced $\mathrm{T}$ cell enrichment in tumor tissue on histologic analysis, and assessment of in vivo transmigration using a mouse air pouch model demonstrated TNC-mediated transmigration of $\mathrm{T}$ cells (81). Pathway analysis in vitro using co-cultures of Jurkat cells with U118MG glioma tumor cells or tumor ECM identified phosphorylation of focal adhesion kinase (FAK) and migrationrelated kinase ERK which was shown to be required for transmigration through a cancer monolayer (81). Interestingly, a potential relationship between tumor ECM constituents and peritumoral edema was also observed in a study by $\mathrm{Qu}$ et al. analyzing the expression of GBM PIEZO1 relative to normal peritumoral tissue in patients; quantification of PIEZO1 expression and image analysis revealed a positive relationship between expression and extent of peritumoral edema where higher expression was observed in patients with severe edema that was defined as an edema index $>3$ (calculated as the ratio of tumor and edema volume to tumor volume) (82). Lastly, it is well known that GBM and the tumor microenvironment present numerous challenges to adequate and effective delivery of therapeutics, and this concept also extends to the physical properties of the tumor and microenvironment. Recent efforts have included innovative strategies to create therapeutics that account for and accommodate the rheological features to engineer adaptive therapeutics (83). Detailed overview of recent trends and advances in overcoming physical barriers to drug delivery can be found elsewhere (84).

\section{Mechanisms of Mechanical Stimuli Transduction in Glioma}

Mechanistic understanding of mechanical stimuli transduction in glioma is lacking, though recent advances have shed some light on the microenvironment and cellular network interactions that underlie the effects of mechanical properties on disease progression. Hubs of mechanical stimuli transduction in gliomas can be generally categorized as either via mechanosensitive ion channels or non-ion channel-based mechanotransduction which encompasses a complex swath of poorly understood signaling pathways including integrin signaling, ligand-mediated signaling 
through interaction with ECM components, or receptor-mediated signaling through interaction with ECM components (85). Here, we briefly summarize representative mechanisms in the evolving framework of glioma mechanotransduction (Table 1).

Three main classes of mechanosensitive ion channels have been implicated in gliomas: PIEZO, TRP, and ENaC (Table 1). Of these, mechanotransduction via PIEZO and TRP channels have been the most well characterized (85). PIEZO1 specifically in glioblastoma serves as an intermediary for a variety of downstream affects including cytoskeletal remodeling, ECM remodeling as well as directly regulating stemness and aggression of cancer cells (71, 85, $88,89)$. Studies have described colocalization of PIEZO1 to regions of cell membrane stress such as those with focal adhesions and signaling via the integrin-FAK pathway $(71,88,89)$. Different subtypes of TRP channels have been implicated in glioma including TRPC1, TRPC6, and TRPM7 (Table 1) (85, 86, 90-94). Similarly, mechanotransduction of physical microenvironmental stimuli through these channels influences downstream regulation of disease progression through effects key cellular structure and function ranging from motility and migration to proliferation and metabolism (86, 90-94). TRP channels in GBM have been associated with activation of Notch signaling and JAK/STAT signaling pathways (94). Ross et al. also identified a potential role for the $\mathrm{ENaC}$ channel which has primarily been shown to regulate cell volume, presumably to facilitate cell motility and migration in complex microenvironments (Table 1) (87). Study of mechanosensitive ion channels has centered largely on those located on the cell membrane, and intracellular or nuclear ion channels in the context of mechanotransduction may represent a new frontier that could improve understanding of downstream signal transduction.

Non-ion channel-based mechanotransduction in glioma is poorly defined and represents an active area of investigation. In recent years, groups have been able to thoroughly characterize a few signal transduction pathways that utilize ligand-mediated, receptor-mediated, and integrin-mediated mechanosensation $(45,76,77,79,95)$. Other studies in this area have focused on defining downstream components of signaling mediators following the initial mechanosensation event, and this has led to the identification YAP/TAZ, PHIP, and MGAT, among others that play a role in glioma stemness and disease progression; however, a unifying mechanism that considers heterogeneity in mechanical properties of the glioma microenvironment as well genetic and treatment-related drivers of plasticity is lacking and requires further investigation (96-104). Kim et al. describe a ligand-mediated mechanotransduction mechanism in gliomas that leverages the interaction between HA that is overexpressed in the ECM and CD44 (Table 1) (77). In this study, the authors observe a temporal component to mechanotransduction where HA-CD44-mediated signaling and consequent enhanced glioma adhesion appears to occur earlier than integrin-mediated signaling and adhesion in glioma cells cultured in modified hydrogels (77). This suggests that early mechanotransduction may occur via an CD44-independent ligand-mediated mechanism whereas the welldescribed ECM-integrin interactions in glioma may occur later. Temporal cues that define these mechanisms as well as the molecular implications of this phenomenon are unknown. Moreover, this finding adds another potential layer of complexity to mechanotransduction mechanisms in glioma in that our current framework does not clearly define whether temporal heterogeneity exists in this mechanism or the previously described mechanisms and whether this is of functional significance. Several groups have described the mechanotransduction scheme and downstream effects of integrin-mediated signaling $(45,79,95)$. TNC has been established as a protein mediator of microenvironment and glioma cell interaction ultimately facilitating and converging on integrin-mediated signaling (70, 76, 105-108). A possible receptor-mediated mechanism for the regulation of mechanosensation has been described by Zhang et al. who show that binding of IL-33 to the ST2 receptor is associated with TNC accumulation and subsequent alteration in the GBM phenotype (Table 1) (76). TNC is an important mediator of mechanical cues in the microenvironment, and these findings suggest that interactions (in this case a receptor-mediated interaction) that alter the availability of such mediators can have implications for mechanotransduction, though further investigation is required to elucidate these potential links.

\section{ROLE OF FLUID MECHANICS IN GLIOMA}

\section{Fluid Shear Stress and Interstitial Fluid Dynamics}

Alteration of brain fluid mechanics in patients with glioma, specifically cerebrospinal fluid (CSF) dynamics is not uncommon (109). This can occur secondary to tumor-related obstruction of natural CSF drainage pathways or dysfunction of CSF resorption. Bloodstream-related fluid shear stress and other fluid stresses has been extensively studied in the context of metastatic cancer as well as other disease processes; however, studies characterizing these forces in glioma and examining the impact on the disease process are sparse. Further investigation in this area may provide a more complete view of the stresses at play

TABLE 1 | Representative Substrates of Mechanical Signal Transduction in Glioma.

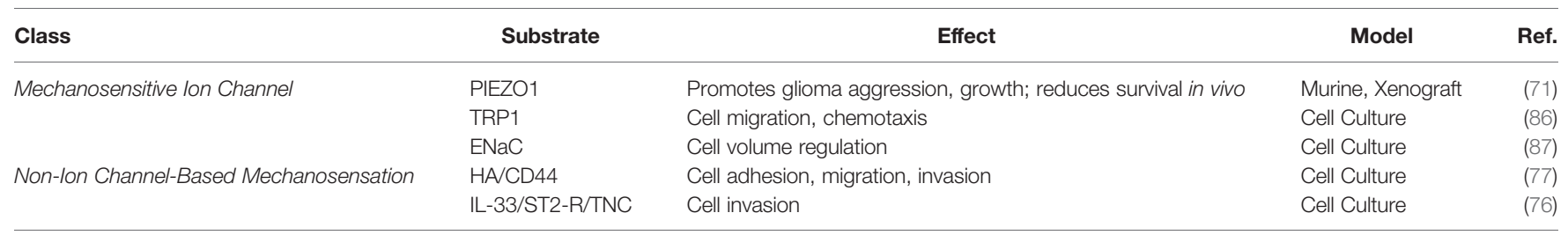

HA, hyaluronic acid; TNC, Tenascin-C; ST2-R, ST2 receptor. 
in the tumor bulk and microenvironment, and early studies suggest that the influence fluid-related stresses may be clinically significant $(85,110-116)$.

Interstitial fluid flow refers to fluid flow generally through a 3dimensional matrix and interstitial fluid pressure (IFP) refers to the biophysical manifestation of the pressure gradient typically between a capillary and a draining lymphatic vessel. In the context of cancer and glioma, an elevated IFP is observed due to increased vessel permeability, i.e., leaky vasculature, secondary to tumor-mediated angiogenesis and dysplastic tumor vessels (Figure 2). The resultant high IFP has several effects on the tumor and tumor microenvironment. First, elevated IFP is transmitted through the tumor milieu and ECM of the glioma subjecting glioma cells and ECM components to various forces including normal force and shear stress. Much of the current understanding of the sequelae of these fluid-based forces in glioma and in the brain in general are extrapolated from studies in other organ systems and disease contexts; this is in part due to the difficulty to accurately measure these stress forces in a complex microenvironment (Figure 2) (117-119). It is presumable that shear stress-mediated deformation of either the cancer cell cytoarchitecture directly or ECM components promotes changes in stemness, migration, and other features of cancer through mechanotransduction as discussed in previous sections, but this is yet to be investigated comprehensively. Qazi et al. examined the effect of simulated fluid shear stress on the migratory activity of glioma cell lines in a modified Boyden chamber (116). The authors demonstrated that both time of exposure to shear stress and magnitude of shear stress diminished migration in two of three cell lines by $92 \%$ and $58 \%$, but the third cell line was not affected by shear stress (116). Quantification of MMP levels showed a concomitant downregulation of active and total MMP with exposure to shear stress that was confirmed with MMP inhibitor assays. Interestingly, the third cell line that was not affected by shear stress also exhibited minimal change in MMP levels. They observed differential migratory activity in the presence of or absence of a TGF- $\alpha$ flow gradient suggesting enhanced cell migration due to a flow-induced chemotaxis-89\%, 566\%, and $101 \%$ enhancement in migratory capacity with TGF- $\alpha$ (116). In similar studies by Li et al. and Namba et al., simulated fluid shear stress applied to U87 glioma cells and BTICs in a microfluidic apparatus produced an increase cellular adhesion strength and differential invasion based on differentiation-less differentiated nestin-positive BTICs tended to invade first under interstitial flow $(115,120)$. In addition to mechanical effects of fluid shear stress and IFP, these forces in various cancer models including have been shown to produce flow-induced gradients of soluble factors in the microenvironments including chemokines that influence directionality and invasion through chemical signaling (Figure 2) $(85,114,118,119,121)$. Although initial studies of interstitial fluid flow and IFP posited a radial IFP emanating from the tumor core outward because of the arrangement of leaky vasculature, Spin echo-MRI analysis by Kingsmore et al. of xenograft mouse glioma tumors revealed heterogenous interstitial flow dynamics $(110,111,122-124)$. They noted a general trend of outward flow of interstitial fluid but observed significant intratumoral heterogeneity in interstitial flow

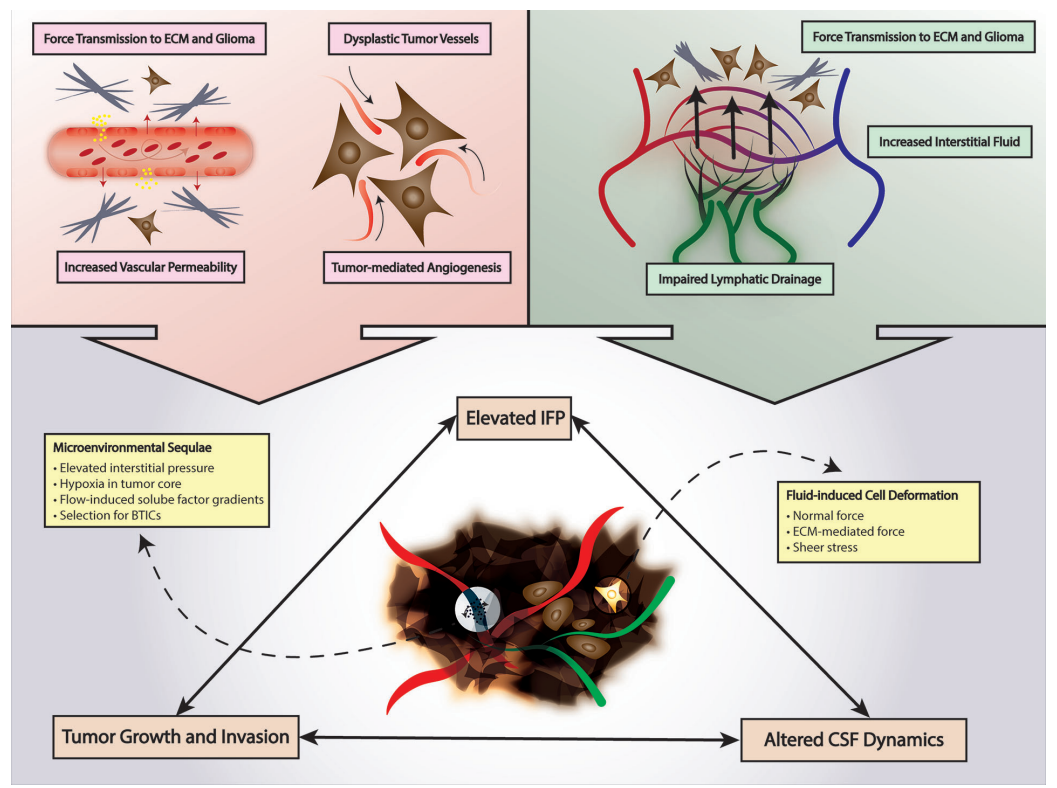

FIGURE 2 | Fluid Mechanics in Glioma. Tumor-mediated angiogenesis and tumor-mediated increased vascular permeability increases interstitial fluid pressure and force transmission to glioma cells and ECM. Lymphatic insufficiency also contributes to increased interstitial fluid pressure. Increased mechanical stimuli from elevated interstitial fluid pressure can promote a tumor environment selective for BTICs and alter CSF dynamics. CSF, cerebrospinal fluid; ECM, extracellular matrix; BTICs, brain tumor-initiating cells; IFP, interstitial fluid pressure. 
velocities which correlated with Evans blue assessment of drainage (111). Taken together with the work by Qazi et al. it is evident that fluid dynamics and the response to local fluid dynamics is not uniform but rather heterogenous $(111,113,116)$. Further investigation of interstitial fluid dynamics and shear stress in glioma will not only improve our understanding of tumoral and microenvironment heterogeneity but also potentially uncover new therapeutic targets that can be modulated to either slow disease progression or perhaps facilitate enhanced drug delivery to sites of disease (113).

\section{Lymphatic Flow Dynamics}

Lymphatic flow dynamics and drainage effectively link microenvironmental properties such as IFP and interstitial fluid flow with the local immunosuppression that is observed in glioma. In brain tumors such as GBM, draining lymphatic vessels of the tumor are typically compromised, and the tumor milieu is inadequately drained. This produces a dual mechanical and immunological effect in the microenvironment. As a result of compromised lymphatics, interstitial fluid accumulates in certain regions of the microenvironment resulting in elevated IFP with its associated mechanical and chemical signaling as previously discussed (Figure 2) (49, 117, 125-127). Secondly, impaired lymphatic drainage from the tumor simultaneously hinders antigen presentation and immune cell recruitment at peripheral sites as well as preventing migration of immune effector cells into the tumor tissue ultimately producing a function immune escape phenomenon (Figure 1) (49, 125127). Studies have demonstrated that pharmacologic restoration of these meningeal lymphatic vessels can sensitize GBM to the host immune response and synergize with immunotherapy effectively $(117,125-128)$. This new class of targeted therapies such as VEGF-C is promising in that it represents a priming of cellular immunotherapy, if successful, can reach infiltrative disease in addition to the primary disease site while concurrently ameliorating malignant mechanical stimuli-induced changes in glioma cells by relieving IFP in areas of impaired lymphatic drainage (49, 125-129).

\section{THERANOSTIC OPPORTUNITIES AND MODELING}

\section{Therapeutic Targets}

Several potential therapeutic targets follow from the present overview of the current understanding of mechanical properties in the glioma microenvironment. Given the crucial role of mechanotransducers such as mechanosensitive ion channels in promoting glioma malignancy, pharmacologic inhibition of signal transduction is attractive. Known inhibitors exist already for some of these channels such as the PIEZO, mechanically gated ion channels; however, non-specificity poses a hurdle as well as the perpetual barrier of achieving effective delivery of any therapeutic to the primary tumor site and invasive BTICs (130-134). Drug pharmacology surrounding the PIEZO channels is still in its infancy, with the majority of studies employing non-specific inhibitors for the purposes of interrogation of channel properties and mechanism of action $(130,133)$. Groups have identified inhibitors of the PIEZO1 and PIEZO2 channel including the polycation ruthenium red, gadolinium, and the peptide GsMTx-4, but these agents are yet to be tested in translational studies. Activators such as Yoda1, Jedil, and Jedi have also been described and these agents have been similarly leveraged to elucidate the mechanism of these channels in the glioma microenvironment $(71,133,135)$. For example, Chen et al. utilize Yodal to further define the relationship between surrounding tissue stiffness and PIEZO1 activity in glioma by showing that beyond a certain level of expression of the channel that is necessary for growth, overactivation of the PIEZO1 channels with Yoda1 do not enhance glioma cell proliferation in vitro (71). As Xiao outlines in his excellent review of the prospects for future therapeutics targeting PIEZO channels, with increasing recognition of the influence of such ion channels on glioma cell phenotype and disease progression, studies directed towards drug discovery and targeted inactivators of mechanosensitive ion channels for clinical application are forthcoming (133). This is supported by the recent elucidation of the crystal structure of such channels that have paved the path for high-throughput, targeted drug development $(136,137)$.

In addition to directly inhibiting mechanical signal transduction, another feasible approach is to target the downstream molecular mechanisms and cellular processes that promote disease progression. One such target is autophagy which has been implicated in treatment resistance in various cancers including GBM (138-140). Recent studies have shown that the process of autophagy is altered by mechanical stimuli and signal transduction primarily through two mechanisms: 1) crosstalk between the shared regulatory proteins in autophagy and mechanical signal transduction pathway or 2) competition for molecular substrates utilized in both processes such as cytoskeletal elements (138). Several common pathways have been described in the literature and include the YAP/TAZ axis, JAK-mediated signal transduction, and the expression of EGFR $(138,141,142)$. Dupont et al. demonstrate in a series of experiments that the transcriptional regulators $\mathrm{YAP} / \mathrm{TAZ}$ are required for transducing mechanical cues from the microenvironment and specifically the elasticity of the ECM (96). Using a stem cell model, they demonstrate that altering ECM stiffness and geometry of the growth substrate differentially regulates both proliferation and cell differentiation in a YAP/TAZ-dependent manner (96). Interestingly, YAP/TAZ is also observed to facilitate the fusion of autophagolysosomes and promote autophagic flux via a downstream protein target, Armus, in a study by Totaro et al. $(101,143)$ When this effect was pharmacologically dampened with autophagy inhibitors or through knockdown of autophagy genes, growth of breast cancer cells in vitro decreased. The opposite effect was observed when YAP/TAZ was overexpressed, and cancer stem cell features such as plasticity as measured by differentiation potential were enhanced (143). This data suggests that the YAP/ TAZ axis may be one of several common pathways between mechanical signal transduction and other aberrant cellular 
processes in cancer that can be simultaneously targeted (143). Taken together, development of therapeutics that interfere with the cytoprotective effects of autophagy in cancer cells may represent a potential treatment modality and serve as an example for identifying points of convergence between mechanical signal transduction and other known processes in cancer progression amenable to therapeutic targeting $(139,140)$.

Alternatively, a therapeutic strategy that alters the mechanical properties of the microenvironment, i.e., manipulating solid stress and fluid stress in the tumor milieu, to engineer an anti-glioma environment may be plausible $(71,83,113,144,145)$. The advantage with such an approach may be the ability to create "smart" or responsive therapies that can either effect mechanical changes locoregionally_in the area of radiographic disease or visible tumor or global changes to target invisible, infiltrative disease $(71,83,113,144,145)$. Many of the studies discussed in this review as well as other studies examining mechanical properties in the cancer microenvironment have used some type of bioengineering approach to create a microenvironmentmimetic substrate to alter the physical forces that are felt by the cell or tissue of interest. In these studies, modulation of the substrate whether it is a $3 \mathrm{D}$ hydrogel or $2 \mathrm{D}$ suspension has resulted in elimination of malignant properties. This concept can be theoretically applied as a treatment whereby engineered materials may be implanted into tumor resection cavities after surgical removal of visible tumor. The material or scaffold could be engineered to respond to chemical and physical stimuli from surrounding brain tissue and to alter local physical properties to promote an anti-glioma microenvironment (114, 116, 146-149). This paradigm requires further study and is yet to be developed for clinical application in glioma. Although bioengineering technologies have evolved to meet these needs, this type of approach is hindered by our current rudimentary understanding of biophysical dynamics and their ramifications in brain cancer and in normal brain. Another approach to achieve the same effect is with the use of pharmacologic agents that can alter mechanical properties in the microenvironment such as with the angiotensin inhibitor Losartan. Chauhan et al. show that in models of breast and prostate cancer, administration of Losartan decreases solid stress in the tumor microenvironment by reducing the production of profibrotic components in the ECM such as collagen and hyaluronan (150). The use of Losartan with the intent to target mechanical properties in the context of brain tumors has not been well-studied, but a Phase 2 clinical trial is currently ongoing within this area (NCT03951142). Even in the absence of therapeutics targeted at tissue mechanics, improved understanding of these properties may pave the way for designing adjunctive therapies that can mitigate the biophysical barriers to other treatments such as drug delivery of conventional chemotherapeutics or effective immune cell infiltration of disease sites and successful immunotherapy-based approaches $(11,23,84,151)$.

\section{Diagnostics}

Unique mechanical properties of glioma and its microenvironment provide a basis for the development of novel diagnostics to address two unmet needs in clinical medicine: 1) accurate identification of infiltrative disease in nervous tissue that is radiographically and microscopically occult and 2) characterization disease heterogeneity and plasticity towards predictive analytics for treatment response or prognosis. Fundamental technologies are available as were described in the studies presented in this review such as MRE and other tools for rheological phenotyping, and these have the potential to be adapted and refined as possible intraoperative adjuncts or as supplements to the conventional imaging obtained for patients with GBM to better guide treatment choices $(57,59,61,62$, 67, 152-154). Briefly, techniques can be categorized based on the substrate assessed-either cells and tissue or more macroscopically a region of the brain (Table 2). MRE as discussed previously, is now a well-studied imaging technique that can characterize tissue stiffness on a global scale in the brain. In this technique, vibrations through the brain are coupled with magnetic resonance imaging (MRI) sequences to create a landscape of tissue stiffness in the brain $(59-62,85,155)$. This is particularly useful when trying to evaluate a mass in the brain as the tissue stiffness within the mass and surrounding regions may offer insights into the tumor type, grade, and propensity for malignant transformation $(59,60,67)$. Investigations are ongoing to optimize the imaging protocols for this technique and to develop iterations that can be used at the point-of-care in the operating room. Intraoperative imaging with this technology could provide information regarding prognostication, response to therapy, growth rate in addition to the other intraoperative imaging tools available currently such as Raman spectroscopy, brain mapping, fluorescence-guidance, and optical coherence tomography (156). Further research is needed to establish and validate MRE as a reliable surrogate for such clinical

TABLE 2 | Representative Methods of Measuring Mechanical Tissue Properties in Glioma.

\begin{tabular}{|c|c|c|c|}
\hline Method & Substrate & Mechanism & Ref. \\
\hline MRE & Brain/Tissue & Stiffness map of ROI & $(59,60,67)$ \\
\hline US & Brain/Tissue & Stiffness based on permeability to ultrasonic waves & (85) \\
\hline SWE & Brain/Tissue & Stiffness based on propagation of ultrasonic waves and tissue displacement & (58) \\
\hline Needle biopsy & Tissue & Solid stress based on tissue deformation & $(54-56,85)$ \\
\hline Serial slices & Tissue & Solid stress based on tissue deformation & $(54-56,85)$ \\
\hline Planar cut & Tissue & Solid stress based on tissue deformation & $(54-56,85)$ \\
\hline AFM & Tissue/Cell & Stiffness based on force measurement between probe and tissue & $(47,85)$ \\
\hline Particle tracking & Tissue/Cell & Live imaging and measurement of particle movement, viscosity measurement & (85) \\
\hline
\end{tabular}

MRE, Magnetic Resonance Elastography; US, ultrasound; SWE, shear wave elastography.

AFM, Atomic Force Microscopy; ROI, region of interest. 
parameters. Ultrasound-based imaging technologies can also be used at the point-of-care, and the two general modalities are traditional ultrasound-based imaging and shear wave elastography (Table 2). In traditional ultrasound, the ultrasound probe can be placed on the tissue of interest during surgery and an interpretation of physical characteristics is made based on the radiolucency of the area of interest. In shear wave elastography, a device is used to measure the propagation of ultrasonic waves through the region of interest and also measure the displacement of the tissue to calculate physical parameters such as shear modulus $(47,58,85)$ (Table 2). Many methods have been described to study the physical properties of cells and tissue, and these include atom force microscopy, particle-tracking techniques, and measurements of tissue deformation (53-55, 110, 122, 124) (Table 2). The latter technique consists of lesioning a piece of tissue typically with a needle biopsy, serial slicing, or a single planar cut and subsequently measuring the magnitude of deformation or displacement of the tissue into the lesioned area. Comprehensive reviews of methodologies used to study mechanical properties in vitro and in vivo can be found elsewhere $(47,85,155)$. Advances in bioengineering in the fields of microfluidics, biomimetics, and hydrogel may also enable the development of high-throughput, point-of-care methods to define disease features such as the mechanophenotype that may aid in clinical-decision making (84, 147, 152, 157).

\section{Preclinical Modeling}

Veritable preclinical models of glioma that accurately recapitulate important aspects of the disease are essential for successful clinical translation of innovative therapeutics. Our growing understanding of all the layers of disease heterogeneity and plasticity is now further complicated by heterogeneity and plasticity of the mechanical properties of glioma and its microenvironment (17, 25, 84, 147, 157, 158). Recent work also highlights clinically significant sexual dimorphism in many facets of the glioma disease process, and this feature of glioma has yet to be studied rigorously from the biophysical perspective which will be an important consideration for therapy development $(20,158-160)$. For example, it is unknown whether sex differences affect solid stress or fluid stress components within a glioma and its microenvironment. Similarly, the influence of other tumor characteristics on the biophysical properties of the tumor microenvironment are also poorly understood. These include factors such as proximity to the cerebrospinal fluid spaces of the brain and the response of tumor to surgical resection and chemoradiation which have been shown to play a role in other processes, but further investigation is needed with regards to mechanical properties $(161,162)$. Once again, the application of bioengineering to model mechanical features of the microenvironment may be several in dissecting mechanisms of

\section{REFERENCES}

1. Ostrom QT, Patil N, Cioffi G, Waite K, Kruchko C, Barnholtz-Sloan JS, et al. CBTRUS Statistical Report: Primary Brain and Other Central Nervous System Tumors Diagnosed in the United States in 2013-2017. Neuro Oncol (2020) 22:iv1-iv96. doi: 10.1093/neuonc/ noaa 200 mechanotransduction, mechanoreciprocity, and plasticity. Current models attempt to incorporate different features of heterogeneity in the tumor microenvironment that may impact its mechanical properties such as varying the composition of the ECM constituents as well as the type of model-3D versus 2D (147). Other groups have recreated heterogeneity in stiffness, elasticity, and soluble factor gradients $(147,149,163,164)$. These models will be crucial for effective drug development because they may offer useful information about pharmacokinetic and pharmacodynamic properties of proposed therapeutics as well as uncover additional therapeutic targets or treatment resistance mechanisms. As new variables are identified that can influence the mechanical properties of the microenvironment, the models that attempt to recapitulate these features will likely continue to become more sophisticated (114, 116, 147-149, 165-167).

\section{CONCLUSION}

The study of mechanical properties in the glioma microenvironment holds considerable promise. Further elucidation of the biophysical features of the microenvironment will enable a more comprehensive understanding of glioma as a disease process, and this may create novel theranostic opportunities while also informing preclinical modeling.

\section{AUTHOR CONTRIBUTIONS}

AB: conceptualization, investigation, writing - original draft, writing - review and editing, visualization, and management. JD: writing - review and editing. RC: writing - review and editing, and supervision. ST: conceptualization, writing - review and editing and supervision. All authors contributed to the article and approved the submitted version.

\section{FUNDING}

ST received funding from the National Institutes of Health (R01CA227838) and a pilot project award from the University of Kansas Cancer Center, NCI support grant P30 CA168524.

\section{ACKNOWLEDGMENTS}

The authors thank Dr. Paul Camarata, MD, Chair, Department of Neurosurgery for supporting the publication costs.

2. ReFaey K, Tripathi S, Grewal SS, Bhargav AG, Quinones DJ, Chaichana KL, et al. Cancer Mortality Rates Increasing vs Cardiovascular Disease Mortality Decreasing in the World: Future Implications. Mayo Clin Proc Innov Qual Outcomes (2021) 5:645-53. doi: 10.1016/j.mayocpiqo.2021.05.005

3. Louis DN, Perry A, Wesseling P, Brat DJ, Cree IA, Figarella-Branger D, et al. The 2021 WHO Classification of Tumors of the Central Nervous System: A Summary. Neuro Oncol (2021) 23:1231-51. doi: 10.1093/neuonc/noab106 
4. Stupp R, Mason WP, van den Bent MJ, Weller M, Fisher B, Taphoorn MJ, et al. Radiotherapy Plus Concomitant and Adjuvant Temozolomide for Glioblastoma. N Engl J Med (2005) 352:987-96. doi: 10.1056/NEJMoa043330

5. Stupp R, Taillibert S, Kanner A, Read W, Steinberg D, Lhermitte B, et al. Effect of Tumor-Treating Fields Plus Maintenance Temozolomide vs Maintenance Temozolomide Alone on Survival in Patients With Glioblastoma: A Randomized Clinical Trial. JAMA (2017) 318:2306-16. doi: 10.1001/jama.2017.18718

6. Sanai N, Berger MS. Surgical Oncology for Gliomas: The State of the Art. Nat Rev Clin Oncol (2018) 15:112-25. doi: 10.1038/nrclinonc.2017.171

7. Lara-Velazquez M, Al-Kharboosh R, Jeanneret S, Vazquez-Ramos C, Mahato D, Tavanaiepour D, et al. Advances in Brain Tumor Surgery for Glioblastoma in Adults. Brain Sci (2017) 7:1-16. doi: 10.3390/brainsci7120166

8. Bhargav AG, Mondal SK, Garcia CA, Green JJ, Quinones-Hinojosa A. Nanomedicine Revisited: Next Generation Therapies for Brain Cancer. Adv Ther-Germany (2020) 3:ARTN 2000118. doi: 10.1002/adtp.202000118

9. Dunn GP, Cloughesy TF, Maus MV, Prins RM, Reardon DA, Sonabend AM, et al. Emerging Immunotherapies for Malignant Glioma: From Immunogenomics to Cell Therapy. Neuro Oncol (2020) 22:1425-38. doi: 10.1093/neuonc/noaa154

10. Lim M, Xia Y, Bettegowda C, Weller M. Current State of Immunotherapy for Glioblastoma. Nat Rev Clin Oncol (2018) 15:422-42. doi: 10.1038/s41571018-0003-5

11. Shah K. Stem Cell-Based Therapies for Tumors in the Brain: Are We There Yet? Neuro Oncol (2016) 18:1066-78. doi: 10.1093/neuonc/now096

12. Stuckey DW, Shah K. Stem Cell-Based Therapies for Cancer Treatment: Separating Hope From Hype. Nat Rev Cancer (2014) 14:683-91. doi: $10.1038 / \mathrm{nrc} 3798$

13. Quinones-Hinojosa A, Sanai N, Soriano-Navarro M, Gonzalez-Perez O, Mirzadeh Z, Gil-Perotin S, et al. Cellular Composition and Cytoarchitecture of the Adult Human Subventricular Zone: A Niche of Neural Stem Cells. $J$ Comp Neurol (2006) 494:415-34. doi: 10.1002/cne.20798

14. Sanai N, Tramontin AD, Quinones-Hinojosa A, Barbaro NM, Gupta N, Kunwar S, et al. Unique Astrocyte Ribbon in Adult Human Brain Contains Neural Stem Cells But Lacks Chain Migration. Nature (2004) 427:740-4. doi: 10.1038/nature02301

15. Lathia J, Liu H, Matei D. The Clinical Impact of Cancer Stem Cells. Oncologist (2020) 25:123-31. doi: 10.1634/theoncologist.2019-0517

16. Lathia JD, Mack SC, Mulkearns-Hubert EE, Valentim CL, Rich JN. Cancer Stem Cells in Glioblastoma. Genes Dev (2015) 29:1203-17. doi: 10.1101/ gad.261982.115

17. Mitchell K, Troike K, Silver DJ, Lathia JD. The Evolution of the Cancer Stem Cell State in Glioblastoma: Emerging Insights Into the Next Generation of Functional Interactions. Neuro Oncol (2021) 23:199-213. doi: 10.1093/ neuonc/noaa259

18. Saygin C, Matei D, Majeti R, Reizes O, Lathia JD. Targeting Cancer Stemness in the Clinic: From Hype to Hope. Cell Stem Cell (2019) 24:25-40. doi: 10.1016/j.stem.2018.11.017

19. Comba A, Faisal SM, Varela ML, Hollon T, Al-Holou WN, Umemura Y, et al. Uncovering Spatiotemporal Heterogeneity of High-Grade Gliomas: From Disease Biology to Therapeutic Implications. Front Oncol (2021) 11:703764. doi: $10.3389 /$ fonc.2021.703764

20. Gimple RC, Bhargava S, Dixit D, Rich JN. Glioblastoma Stem Cells: Lessons From the Tumor Hierarchy in a Lethal Cancer. Genes Dev (2019) 33:591609. doi: 10.1101/gad.324301.119

21. Prager BC, Bhargava S, Mahadev V, Hubert CG, Rich JN. Glioblastoma Stem Cells: Driving Resilience Through Chaos. Trends Cancer (2020) 6:223-35. doi: 10.1016/j.trecan.2020.01.009

22. Fecci PE, Sampson JH. The Current State of Immunotherapy for Gliomas: An Eye Toward the Future. J Neurosurg (2019) 131:657-66. doi: 10.3171/ 2019.5.JNS181762

23. Yeini E, Ofek P, Albeck N, Ajamil DR, Neufeld L, Eldar-Boock A, et al. Targeting Glioblastoma: Advances in Drug Delivery and Novel Therapeutic Approaches. Adv Ther-Germany (2021) 4:ARTN 2000124. doi: 10.1002/ adtp. 202000124

24. Ma YF, Yang ZG, Huntoon K, Jiang W, Kim BYS. Advanced Immunotherapy Approaches for Glioblastoma. Adv Ther-Germany (2021) 4:ARTN 2100046. doi: 10.1002/adtp.202100046
25. Lathia JD, Heddleston JM, Venere M, Rich JN. Deadly Teamwork: Neural Cancer Stem Cells and the Tumor Microenvironment. Cell Stem Cell (2011) 8:482-5. doi: 10.1016/j.stem.2011.04.013

26. Bonavia R, Inda MM, Cavenee WK, Furnari FB. Heterogeneity Maintenance in Glioblastoma: A Social Network. Cancer Res (2011) 71:4055-60. doi: 10.1158/0008-5472.CAN-11-0153

27. Gilbertson RJ, Rich JN. Making a Tumour's Bed: Glioblastoma Stem Cells and the Vascular Niche. Nat Rev Cancer (2007) 7:733-6. doi: 10.1038/ $\operatorname{nrc} 2246$

28. Andersen BM, Faust Akl C, Wheeler MA, Chiocca EA, Reardon DA, Quintana FJ. Glial and Myeloid Heterogeneity in the Brain Tumour Microenvironment. Nat Rev Cancer (2021) 23:199-213. doi: 10.1038/s41568-021-00397-3

29. Barnes JM, Przybyla L, Weaver VM. Tissue Mechanics Regulate Brain Development, Homeostasis and Disease. J Cell Sci (2017) 130:71-82. doi: $10.1242 /$ jcs. 191742

30. Kaushik S, Persson AI. Unlocking the Dangers of a Stiffening Brain. Neuron (2018) 100:763-5. doi: 10.1016/j.neuron.2018.11.011

31. Iwashita M, Kataoka N, Toida K, Kosodo Y. Systematic Profiling of Spatiotemporal Tissue and Cellular Stiffness in the Developing Brain. Development (2014) 141:3793-8. doi: 10.1242/dev.109637

32. Heisenberg CP, Bellaiche Y. Forces in Tissue Morphogenesis and Patterning. Cell (2013) 153:948-62. doi: 10.1016/j.cell.2013.05.008

33. Sawamoto K, Wichterle H, Gonzalez-Perez O, Cholfin JA, Yamada M, Spassky N, et al. New Neurons Follow the Flow of Cerebrospinal Fluid in the Adult Brain. Science (2006) 311:629-32. doi: 10.1126/science.1119133

34. Alvarez-Buylla A, Lim DA. For the Long Run: Maintaining Germinal Niches in the Adult Brain. Neuron (2004) 41:683-6. doi: 10.1016/s0896-6273(04) 00111-4

35. Saha K, Keung AJ, Irwin EF, Li Y, Little L, Schaffer DV, et al. Substrate Modulus Directs Neural Stem Cell Behavior. Biophys J (2008) 95:4426-38. doi: $10.1529 /$ biophysj.108.132217

36. Georges PC, Miller WJ, Meaney DF, Sawyer ES, Janmey PA. Matrices With Compliance Comparable to That of Brain Tissue Select Neuronal Over Glial Growth in Mixed Cortical Cultures. Biophys J (2006) 90:3012-8. doi: 10.1529/biophysj.105.073114

37. Leipzig ND, Shoichet MS. The Effect of Substrate Stiffness on Adult Neural Stem Cell Behavior. Biomaterials (2009) 30:6867-78. doi: 10.1016/ j.biomaterials.2009.09.002

38. Guo J, Bertalan G, Meierhofer D, Klein C, Schreyer S, Steiner B, et al. Brain Maturation is Associated With Increasing Tissue Stiffness and Decreasing Tissue Fluidity. Acta Biomater (2019) 99:433-42. doi: 10.1016/ j.actbio.2019.08.036

39. Chang EH, Adorjan I, Mundim MV, Sun B, Dizon MLV, Szele FG. Traumatic Brain Injury Activation of the Adult Subventricular Zone Neurogenic Niche. Front Neurosci (2016) 10:332. doi: 10.3389/fnins.2016.00332

40. Laywell ED, Dorries U, Bartsch U, Faissner A, Schachner M, Steindler DA, et al. Enhanced Expression of the Developmentally Regulated Extracellular Matrix Molecule Tenascin Following Adult Brain Injury. Proc Natl Acad Sci USA (1992) 89:2634-8. doi: 10.1073/pnas.89.7.2634

41. Murphy MC, Jones DT, Jack CR Jr, Glaser KJ, Senjem ML, Manduca A, et al. Decreased Brain Stiffness in Alzheimer's Disease Determined by Magnetic Resonance Elastography. J Magn Reson Imaging (2011) 34:494-8. doi: $10.1002 / j m r i .22707$

42. Murphy MC, Jones DT, Jack CR Jr, Glaser KJ, Senjem ML, Manduca A, et al. Regional Brain Stiffness Changes Across the Alzheimer's Disease Spectrum. NeuroImage Clin (2016) 10:283-90. doi: 10.1016/j.nicl.2015.12.007

43. Lau LW, Cua R, Keough MB, Haylock-Jacobs S, Yong VW. Pathophysiology of the Brain Extracellular Matrix: A New Target for Remyelination. Nat Rev Neurosci (2013) 14:722-9. doi: 10.1038/nrn3550

44. Bonneh-Barkay D, Wiley CA. Brain Extracellular Matrix in Neurodegeneration. Brain Pathol (2009) 19:573-85. doi: 10.1111/j.17503639.2008.00195. $\mathrm{x}$

45. Levental KR, Yu H, Kass L, Lakins JN, Egeblad M, Erler JT, et al. Matrix Crosslinking Forces Tumor Progression by Enhancing Integrin Signaling. Cell (2009) 139:891-906. doi: 10.1016/j.cell.2009.10.027

46. Katira P, Bonnecaze RT, Zaman MH. Modeling the Mechanics of Cancer: Effect of Changes in Cellular and Extra-Cellular Mechanical Properties. Front Oncol (2013) 3:145. doi: 10.3389/fonc.2013.00145 
47. Kumar S, Weaver VM. Mechanics, Malignancy, and Metastasis: The Force Journey of a Tumor Cell. Cancer Metastasis Rev (2009) 28:113-27. doi: 10.1007/s10555-008-9173-4

48. Tung JC, Barnes JM, Desai SR, Sistrunk C, Conklin MW, Schedin P, et al. Tumor Mechanics and Metabolic Dysfunction. Free Radic Biol Med (2015) 79:269-80. doi: 10.1016/j.freeradbiomed.2014.11.020

49. Swartz MA, Lund AW. Lymphatic and Interstitial Flow in the Tumour Microenvironment: Linking Mechanobiology With Immunity. Nat Rev Cancer (2012) 12:210-9. doi: 10.1038/nrc3186

50. Quail DF, Joyce JA. Microenvironmental Regulation of Tumor Progression and Metastasis. Nat Med (2013) 19:1423-37. doi: 10.1038/nm.3394

51. Mohammadi H, Sahai E. Mechanisms and Impact of Altered Tumour Mechanics. Nat Cell Biol (2018) 20:766-74. doi: 10.1038/s41556-018-0131-2

52. Nia HT, Munn LL, Jain RK. Physical Traits of Cancer. Science (2020) 370:1-9. doi: 10.1126/science.aaz0868

53. Stylianopoulos T, Martin JD, Chauhan VP, Jain SR, Diop-Frimpong B, Bardeesy N, et al. Causes, Consequences, and Remedies for Growth-Induced Solid Stress in Murine and Human Tumors. Proc Natl Acad Sci USA (2012) 109:15101-8. doi: 10.1073/pnas.1213353109

54. Nia HT, Liu H, Seano G, Datta M, Jones D, Rahbari N, et al. Solid Stress and Elastic Energy as Measures of Tumour Mechanopathology. Nat BioMed Eng (2016) 1:1-11. doi: 10.1038/s41551-016-0004

55. Stylianopoulos T, Martin JD, Snuderl M, Mpekris F, Jain SR, Jain RK, et al. Coevolution of Solid Stress and Interstitial Fluid Pressure in Tumors During Progression: Implications for Vascular Collapse. Cancer Res (2013) 73:383341. doi: 10.1158/0008-5472.CAN-12-4521

56. Seano G, Nia HT, Emblem KE, Datta M, Ren J, Krishnan S, et al. Solid Stress in Brain Tumours Causes Neuronal Loss and Neurological Dysfunction and can be Reversed by Lithium. Nat BioMed Eng (2019) 3:230-45. doi: 10.1038/ s41551-018-0334-7

57. Xu L, Lin Y, Han JC, Xi ZN, Shen H, Gao PY, et al. Magnetic Resonance Elastography of Brain Tumors: Preliminary Results. Acta Radiol (2007) 48:327-30. doi: 10.1080/02841850701199967

58. Chauvet D, Imbault M, Capelle L, Demene C, Mossad M, Karachi C, et al. In Vivo Measurement of Brain Tumor Elasticity Using Intraoperative Shear Wave Elastography. Ultraschall Med (2016) 37:584-90. doi: 10.1055/s-00341399152

59. Reiss-Zimmermann M, Streitberger KJ, Sack I, Braun J, Arlt F, Fritzsch D, et al. High Resolution Imaging of Viscoelastic Properties of Intracranial Tumours by Multi-Frequency Magnetic Resonance Elastography. Clin Neuroradiol (2015) 25:371-8. doi: 10.1007/s00062-014-0311-9

60. Streitberger KJ, Reiss-Zimmermann M, Freimann FB, Bayerl S, Guo J, Arlt F, et al. High-Resolution Mechanical Imaging of Glioblastoma by Multifrequency Magnetic Resonance Elastography. PloS One (2014) 9: e110588. doi: 10.1371/journal.pone.0110588

61. Simon M, Guo J, Papazoglou S, Scholand-Engler H, Erdmann C, Melchert $\mathrm{U}$, et al. Non-Invasive Characterization of Intracranial Tumors by Magnetic Resonance Elastography. New J Phys (2013) 15:Artn 085024. doi: 10.1088/ $1367-2630 / 15 / 8 / 085024$

62. Schregel K, Nazari N, Nowicki MO, Palotai M, Lawler SE, Sinkus R, et al. Characterization of Glioblastoma in an Orthotopic Mouse Model With Magnetic Resonance Elastography. NMR BioMed (2018) 31:e3840. doi: $10.1002 / \mathrm{nbm} .3840$

63. Reinhard J, Brosicke N, Theocharidis U, Faissner A. The Extracellular Matrix Niche Microenvironment of Neural and Cancer Stem Cells in the Brain. Int J Biochem Cell Biol (2016) 81:174-83. doi: 10.1016/ j.biocel.2016.05.002

64. Ruoslahti E. Brain Extracellular Matrix. Glycobiology (1996) 6:489-92. doi: 10.1093/glycob/6.5.489

65. Quail DF, Joyce JA. The Microenvironmental Landscape of Brain Tumors. Cancer Cell (2017) 31:326-41. doi: 10.1016/j.ccell.2017.02.009

66. Pogoda K, Chin L, Georges PC, Byfield FJ, Bucki R, Kim R, et al. Compression Stiffening of Brain and its Effect on Mechanosensing by Glioma Cells. New J Phys (2014) 16:75002. doi: 10.1088/1367-2630/16/7/ 075002

67. Streitberger KJ, Lilaj L, Schrank F, Braun J, Hoffmann KT, Reiss-Zimmermann $\mathrm{M}$, et al. How Tissue Fluidity Influences Brain Tumor Progression. P Natl Acad Sci USA (2020) 117:128-34. doi: 10.1073/pnas.1913511116
68. Pope WB, Kim HJ, Huo J, Alger J, Brown MS, Gjertson D, et al. Recurrent Glioblastoma Multiforme: ADC Histogram Analysis Predicts Response to Bevacizumab Treatment. Radiology (2009) 252:182-9. doi: 10.1148/ radiol.2521081534

69. Pope WB, Mirsadraei L, Lai A, Eskin A, Qiao J, Kim HJ, et al. Differential Gene Expression in Glioblastoma Defined by ADC Histogram Analysis: Relationship to Extracellular Matrix Molecules and Survival. AJNR Am J Neuroradiol (2012) 33:1059-64. doi: 10.3174/ajnr.A2917

70. Miroshnikova YA, Mouw JK, Barnes JM, Pickup MW, Lakins JN, Kim Y, et al. Tissue Mechanics Promote IDH1-Dependent HIF1alpha-Tenascin C Feedback to Regulate Glioblastoma Aggression. Nat Cell Biol (2016) 18:1336-45. doi: 10.1038/ncb3429

71. Chen X, Wanggou S, Bodalia A, Zhu M, Dong W, Fan JJ, et al. A Feedforward Mechanism Mediated by Mechanosensitive Ion Channel PIEZO1 and Tissue Mechanics Promotes Glioma Aggression. Neuron (2018) 100:799-815 e797. doi: 10.1016/j.neuron.2018.09.046

72. Ulrich TA, de Juan Pardo EM, Kumar S. The Mechanical Rigidity of the Extracellular Matrix Regulates the Structure, Motility, and Proliferation of Glioma Cells. Cancer Res (2009) 69:4167-74. doi: 10.1158/0008-5472.CAN08-4859

73. Umesh V, Rape AD, Ulrich TA, Kumar S. Microenvironmental Stiffness Enhances Glioma Cell Proliferation by Stimulating Epidermal Growth Factor Receptor Signaling. PloS One (2014) 9:e101771. doi: 10.1371/ journal.pone.0101771

74. Pajic M, Herrmann D, Vennin C, Conway JRW, Chin VT, Jonhsson A-KE, et al. The Dynamics of Rho GTPase Signaling and Implications for Targeting Cancer and the Tumor Microenvironment. Small GTPases (2015) 6:123-33. doi: 10.4161/21541248.2014.973749

75. Northey JJ, Przybyla L, Weaver VM. Tissue Force Programs Cell Fate and Tumor Aggression. Cancer Discovery (2017) 7:1224-37. doi: 10.1158/21598290.Cd-16-0733

76. Zhang JF, Tao T, Wang K, Zhang GX, Yan Y, Lin HR, et al. IL-33/ST2 Axis Promotes Glioblastoma Cell Invasion by Accumulating Tenascin-C. Sci Rep (2019) 9:20276. doi: 10.1038/s41598-019-56696-1

77. Kim Y, Kumar S. CD44-Mediated Adhesion to Hyaluronic Acid Contributes to Mechanosensing and Invasive Motility. Mol Cancer Res (2014) 12:141629. doi: 10.1158/1541-7786.MCR-13-0629

78. Tao B, Song Y, Wu Y, Yang X, Peng T, Peng L, et al. Matrix Stiffness Promotes Glioma Cell Stemness by Activating BCL9L/Wnt/beta-Catenin Signaling. Aging (Albany NY) (2021) 13:5284-96. doi: 10.18632/aging.202449

79. Barnes JM, Kaushik S, Bainer RO, Sa JK, Woods EC, Kai F, et al. A TensionMediated Glycocalyx-Integrin Feedback Loop Promotes Mesenchymal-Like Glioblastoma. Nat Cell Biol (2018) 20:1203-14. doi: 10.1038/s41556-018-0183-3

80. Joyce JA, Fearon DT. T Cell Exclusion, Immune Privilege, and the Tumor Microenvironment. Science (2015) 348:74-80. doi: 10.1126/science.aaa6204

81. Huang JY, Cheng YJ, Lin YP, Lin HC, Su CC, Juliano R, et al. Extracellular Matrix of Glioblastoma Inhibits Polarization and Transmigration of T Cells: The Role of Tenascin-C in Immune Suppression. J Immunol (2010) 185:1450-9. doi: 10.4049/jimmunol.0901352

82. Qu S, Hu T, Qiu O, Su Y, Gu J, Xia Z, et al. Effect of Piezol Overexpression on Peritumoral Brain Edema in Glioblastomas. AJNR Am J Neuroradiol (2020) 41:1423-9. doi: 10.3174/ajnr.A6638

83. Parkins CC, MCAbee JH, Ruff L, Wendler A, Mair R, Gilbertson RJ, et al. Mechanically Matching the Rheological Properties of Brain Tissue for DrugDelivery in Human Glioblastoma Models. Biomaterials (2021) 276:120919. doi: 10.1016/j.biomaterials.2021.120919

84. Stylianopoulos T, Munn LL, Jain RK. Reengineering the Physical Microenvironment of Tumors to Improve Drug Delivery and Efficacy: From Mathematical Modeling to Bench to Bedside. Trends Cancer (2018) 4:292-319. doi: 10.1016/j.trecan.2018.02.005

85. Momin A, Bahrampour S, Min HK, Chen X, Wang X, Sun Y, et al. Channeling Force in the Brain: Mechanosensitive Ion Channels Choreograph Mechanics and Malignancies. Trends Pharmacol Sci (2021) 42:367-84. doi: 10.1016/j.tips.2021.02.006

86. Bomben VC, Turner KL, Barclay TT, Sontheimer H. Transient Receptor Potential Canonical Channels are Essential for Chemotactic Migration of Human Malignant Gliomas. J Cell Physiol (2011) 226:1879-88. doi: 10.1002/ jcp. 22518 
87. Ross SB, Fuller CM, Bubien JK, Benos DJ. Amiloride-Sensitive Na+ Channels Contribute to Regulatory Volume Increases in Human Glioma Cells. Am J Physiol Cell Physiol (2007) 293:C1181-1185. doi: 10.1152/ ajpcell.00066.2007

88. De Felice D, Alaimo A. Mechanosensitive Piezo Channels in Cancer: Focus on Altered Calcium Signaling in Cancer Cells and in Tumor Progression. Cancers (Basel) (2020) 12:1-14. doi: 10.3390/cancers12071780

89. Zhou W, Liu X, van Wijnbergen JWM, Yuan L, Liu Y, Zhang C, et al. Identification of PIEZO1 as a Potential Prognostic Marker in Gliomas. Sci Rep (2020) 10:16121. doi: 10.1038/s41598-020-72886-8

90. Chigurupati S, Venkataraman R, Barrera D, Naganathan A, Madan M, Paul L, et al. Receptor Channel TRPC6 is a Key Mediator of Notch-Driven Glioblastoma Growth and Invasiveness. Cancer Res (2010) 70:418-27. doi: 10.1158/0008-5472.CAN-09-2654

91. Cuddapah VA, Turner KL, Sontheimer H. Calcium Entry via TRPC1 Channels Activates Chloride Currents in Human Glioma Cells. Cell Calcium (2013) 53:187-94. doi: 10.1016/j.ceca.2012.11.013

92. Ding X, He Z, Zhou K, Cheng J, Yao H, Lu D, et al. Essential Role of TRPC6 Channels in G2/M Phase Transition and Development of Human Glioma. J Natl Cancer Inst (2010) 102:1052-68. doi: 10.1093/ jnci/djq217

93. Lepannetier S, Zanou N, Yerna X, Emeriau N, Dufour I, Masquelier J, et al. Sphingosine-1-Phosphate-Activated TRPC1 Channel Controls Chemotaxis of Glioblastoma Cells. Cell Calcium (2016) 60:373-83. doi: 10.1016/ j.ceca.2016.09.002

94. Liu M, Inoue K, Leng T, Guo S, Xiong ZG. TRPM7 Channels Regulate Glioma Stem Cell Through STAT3 and Notch Signaling Pathways. Cell Signal (2014) 26:2773-81. doi: 10.1016/j.cellsig.2014.08.020

95. Lathia JD, Gallagher J, Heddleston JM, Wang J, Eyler CE, MacSwords J, et al. Integrin Alpha 6 Regulates Glioblastoma Stem Cells. Cell Stem Cell (2010) 6:421-32. doi: 10.1016/j.stem.2010.02.018

96. Dupont S, Morsut L, Aragona M, Enzo E, Giulitti S, Cordenonsi M, et al. Role of YAP/TAZ in Mechanotransduction. Nature (2011) 474:179-83. doi: $10.1038 /$ nature 10137

97. Elosegui-Artola A, et al. Force Triggers YAP Nuclear Entry by Regulating Transport Across Nuclear Pores. Cell (2017) 171:1397-1410 e1314. doi: 10.1016/j.cell.2017.10.008

98. Halder G, Dupont S, Piccolo S. Transduction of Mechanical and Cytoskeletal Cues by YAP and TAZ. Nat Rev Mol Cell Biol (2012) 13:591-600. doi: $10.1038 / \mathrm{nrm} 3416$

99. Novev JK, Heltberg ML, Jensen MH, Doostmohammadi A. Spatiotemporal Model of Cellular Mechanotransduction via Rho and YAP. Integr Biol (Camb) (2021) 13:197-209. doi: 10.1093/intbio/zyab012

100. Panciera T, Azzolin L, Cordenonsi M, Piccolo S. Mechanobiology of YAP and TAZ in Physiology and Disease. Nat Rev Mol Cell Biol (2017) 18:758-70. doi: $10.1038 / \mathrm{nrm} .2017 .87$

101. Totaro A, Panciera T, Piccolo S. YAP/TAZ Upstream Signals and Downstream Responses. Nat Cell Biol (2018) 20:888-99. doi: 10.1038/ s41556-018-0142-z

102. Zhang Y, Xie P, Wang X, Pan P, Wang Y, Zhang H, et al. YAP Promotes Migration and Invasion of Human Glioma Cells. J Mol Neurosci (2018) 64:262-72. doi: 10.1007/s12031-017-1018-6

103. de Semir D, Bezrookove V, Nosrati M, Scanlon KR, Singer E, Judkins J, et al. PHIP Drives Glioblastoma Motility and Invasion by Regulating the Focal Adhesion Complex. Proc Natl Acad Sci USA (2020) 117:9064-73. doi: 10.1073/pnas.1914505117

104. Marhuenda E, Fabre C, Zhang C, Martin-Fernandez M, Iskratsch T, Saleh A, et al. Glioma Stem Cells Invasive Phenotype at Optimal Stiffness is Driven by MGAT5 Dependent Mechanosensing. J Exp Clin Cancer Res (2021) 40:139. doi: 10.1186/s13046-021-01925-7

105. Sivasankaran B, et al. Tenascin-C is a Novel RBPJkappa-Induced Target Gene for Notch Signaling in Gliomas. Cancer Res (2009) 69:458-65. doi: 10.1158/0008-5472.CAN-08-2610

106. Xia S, Lal B, Tung B, Wang S, Goodwin CR, Laterra J, et al. Tumor Microenvironment Tenascin-C Promotes Glioblastoma Invasion and Negatively Regulates Tumor Proliferation. Neuro Oncol (2016) 18:507-17. doi: 10.1093/neuonc/nov171
107. Garcion E, Halilagic A, Faissner Affrench-Constant, C. Generation of an Environmental Niche for Neural Stem Cell Development by the Extracellular Matrix Molecule Tenascin C. Development (2004) 131:3423-32. doi: 10.1242/dev.01202

108. Sarkar S, Nuttall RK, Liu S, Edwards DR, Yong VW. Tenascin-C Stimulates Glioma Cell Invasion Through Matrix Metalloproteinase-12. Cancer Res (2006) 66:11771-80. doi: 10.1158/0008-5472.CAN-05-0470

109. Bir SC, Patra DP, Maiti TK, Sun H, Guthikonda B, Notarianni C, et al. Epidemiology of Adult-Onset Hydrocephalus: Institutional Experience With 2001 Patients. Neurosurg Focus (2016) 41:E5. doi: 10.3171/2016.7. FOCUS16188

110. Boucher Y, Baxter LT, Jain RK. Interstitial Pressure Gradients in TissueIsolated and Subcutaneous Tumors: Implications for Therapy. Cancer Res (1990) 50:4478-84. doi: 10.1158/0008-5472.CAN-05-0470

111. Kingsmore KM, Vaccari A, Abler D, Cui SX, Epstein FH, Rockne RC, et al. MRI Analysis to Map Interstitial Flow in the Brain Tumor Microenvironment. APL Bioeng (2018) 2:1-12. doi: 10.1063/1.5023503

112. Mitchell MJ, King MR. Computational and Experimental Models of Cancer Cell Response to Fluid Shear Stress. Front Oncol (2013) 3:44. doi: 10.3389/ fonc.2013.00044

113. Munson JM. Interstitial Fluid Flow Under the Microscope: Is it a Future Drug Target for High Grade Brain Tumours Such as Glioblastoma? Expert Opin Ther Targets (2019) 6:1-13. doi: 10.1080/14728222.2019.1647167

114. Munson JM, Bellamkonda RV, Swartz MA. Interstitial Flow in a 3D Microenvironment Increases Glioma Invasion by a CXCR4-Dependent Mechanism. Cancer Res (2013) 73:1536-46. doi: 10.1158/0008-5472.CAN$12-2838$

115. Namba N, Chonan Y, Nunokawa T, Sampetrean O, Saya H, Sudo R, et al. Heterogeneous Glioma Cell Invasion Under Interstitial Flow Depending on Their Differentiation Status. Tissue Eng Part A (2021) 27:467-78. doi: 10.1089/ten.TEA.2020.0280

116. Qazi H, Shi ZD, Tarbell JM. Fluid Shear Stress Regulates the Invasive Potential of Glioma Cells via Modulation of Migratory Activity and Matrix Metalloproteinase Expression. PloS One (2011) 6:e20348. doi: 10.1371/journal.pone.0020348

117. Ahn JH, Cho H, Kim J-H, Kim SH, Ham J-S, Park I, et al. Meningeal Lymphatic Vessels at the Skull Base Drain Cerebrospinal Fluid. Nature (2019) 572:62-6. doi: 10.1038/s41586-019-1419-5

118. Butcher DT, Alliston T, Weaver VM. A Tense Situation: Forcing Tumour Progression. Nat Rev Cancer (2009) 9:108-22. doi: 10.1038/nrc2544

119. DuFort CC, Paszek MJ, Weaver VM. Balancing Forces: Architectural Control of Mechanotransduction. Nat Rev Mol Cell Biol (2011) 12:308-19. doi: $10.1038 / \mathrm{nrm} 3112$

120. Li W, Mao S, Khan M, Zhang Q, Huang Q, Feng S, et al. Responses of Cellular Adhesion Strength and Stiffness to Fluid Shear Stress During Tumor Cell Rolling Motion. ACS Sens (2019) 4:1710-5. doi: 10.1021/acssensors.9b00678

121. Polacheck WJ, Charest JL, Kamm RD. Interstitial Flow Influences Direction of Tumor Cell Migration Through Competing Mechanisms. Proc Natl Acad Sci USA (2011) 108:11115-20. doi: 10.1073/pnas.1103581108

122. Netti PA, Roberge S, Boucher Y, Baxter LT, Jain RK. Effect of Transvascular Fluid Exchange on Pressure-Flow Relationship in Tumors: A Proposed Mechanism for Tumor Blood Flow Heterogeneity. Microvasc Res (1996) 52:27-46. doi: 10.1006/mvre.1996.0041

123. Rutkowski JM, Swartz MA. A Driving Force for Change: Interstitial Flow as a Morphoregulator. Trends Cell Biol (2007) 17:44-50. doi: 10.1016/ j.tcb.2006.11.007

124. Boucher Y, Jain RK. Microvascular Pressure is the Principal Driving Force for Interstitial Hypertension in Solid Tumors: Implications for Vascular Collapse. Cancer Res (1992) 52:5110-4. doi: 10.1016/j.tcb.2006.11.007

125. Hu X, Deng Q, Ma L, Li Q, Chen Y, Liao Y, et al. Meningeal Lymphatic Vessels Regulate Brain Tumor Drainage and Immunity. Cell Res (2020) 30:229-43. doi: 10.1038/s41422-020-0287-8

126. Kanamori M, Kipnis J. Meningeal Lymphatics "Drain" Brain Tumors. Cell Res (2020) 30:191-2. doi: 10.1038/s41422-020-0286-9

127. Song E, Mao T, Dong H, Boisserand LSB, Antila S, Bosenberg M, et al. VEGF-C-Driven Lymphatic Drainage Enables Immunosurveillance of Brain Tumours. Nature (2020) 577:689-94. doi: 10.1038/s41586-019-1912-x 
128. Dafni H, Israely T, Bhujwalla ZM, Benjamin LE, Neeman M. Overexpression of Vascular Endothelial Growth Factor 165 Drives Peritumor Interstitial Convection and Induces Lymphatic Drain: Magnetic Resonance Imaging, Confocal Microscopy, and Histological Tracking of Triple-Labeled Albumin. Cancer Res (2002) 62:6731-9. doi: 10.1038/s41586-019-1912-x

129. Louveau A, Herz J, Alme MN, Salvador AF, Dong MQ, Viar KE, et al. CNS Lymphatic Drainage and Neuroinflammation are Regulated by Meningeal Lymphatic Vasculature. Nat Neurosci (2018) 21:1380-91. doi: 10.1038/ s41593-018-0227-9

130. Bae C, Sachs F, Gottlieb PA. The Mechanosensitive Ion Channel Piezol is Inhibited by the Peptide Gsmtx4. Biochemistry (2011) 50:6295-300. doi: $10.1021 /$ bi200770q

131. Coste B, Mathur J, Schmidt M, Eraley TJ, Ranade S, Petrus MJ, et al. Piezo1 and Piezo2 are Essential Components of Distinct Mechanically Activated Cation Channels. Science (2010) 330:55-60. doi: 10.1126/science.1193270

132. Syeda R, Xu J, Dubin AE, Coste B, Mathur J. Chemical Activation of the Mechanotransduction Channel Piezol. Elife (2015) 4:6295-300. doi: 10.7554/eLife.07369

133. Xiao B. Levering Mechanically Activated Piezo Channels for Potential Pharmacological Intervention. Annu Rev Pharmacol Toxicol (2020) 60:195-218. doi: 10.1146/annurev-pharmtox-010919-023703

134. Gnanasambandam R, Ghatak C, Yasmann A, Nishizawa K, Sachs F, Ladokhin AS, et al. GsMTx4: Mechanism of Inhibiting Mechanosensitive Ion Channels. Biophys J (2017) 112:31-45. doi: 10.1016/j.bpj.2016.11.013

135. Wang Y, Chi S, Guo H, Li G, Wang L, Zhao Q, et al. A Lever-Like Transduction Pathway for Long-Distance Chemical- and Mechano-Gating of the Mechanosensitive Piezol Channel. Nat Commun (2018) 9:1300. doi: 10.1038/s41467-018-03570-9

136. Zhao Q, Zhou H, Chi S, Wang Y, Wang J, Geng J, et al. Structure and Mechanogating Mechanism of the Piezol Channel. Nature (2018) 554:48792. doi: 10.1038/nature25743

137. Saotome K, Murthy SE, Kefauver JM, Whitwam T, Patapoutian. Structure of the Mechanically Activated Ion Channel Piezo1. Nature (2018) 554:481-6. doi: $10.1038 /$ nature 25453

138. Hernandez-Caceres MP, Munoz L, Pradenas JM, Pena F, Lagos P, Aceiton P, et al. Mechanobiology of Autophagy: The Unexplored Side of Cancer. Front Oncol (2021) 11:632956. doi: 10.3389/fonc.2021.632956

139. Kanzawa T, Germano IM, Komata T, Ito H, Kondo Y, Kondo S. Role of Autophagy in Temozolomide-Induced Cytotoxicity for Malignant Glioma Cells. Cell Death Differ (2004) 11:448-57. doi: 10.1038/sj.cdd.4401359

140. Ulasov I, Fares J, Timashev P, Lesniak MS. Editing Cytoprotective Autophagy in Glioma: An Unfulfilled Potential for Therapy. Trends $\mathrm{Mol}$ Med (2020) 26:252-62. doi: 10.1016/j.molmed.2019.11.001

141. Pavel M, Renna M, Park SJ, Menzies FM, Ricketts T, Füllgrabe J, et al. Contact Inhibition Controls Cell Survival and Proliferation via YAP/TAZAutophagy Axis. Nat Commun (2018) 9:2961. doi: 10.1038/s41467-01805388-x

142. Jutten B, Keulers TG, Schaaf MBE, Savelkouls K, Theys J, Span PN, et al. EGFR Overexpressing Cells and Tumors are Dependent on Autophagy for Growth and Survival. Radiother Oncol (2013) 108:479-83. doi: 10.1016/ j.radonc.2013.06.033

143. Totaro A, Zhuang Q, Panciera T, Battilana G, Azzolin L, Brumana G, et al. Cell Phenotypic Plasticity Requires Autophagic Flux Driven by YAP/TAZ Mechanotransduction. Proc Natl Acad Sci USA (2019) 116:17848-57. doi: $10.1073 /$ pnas.1908228116

144. Sivakumar H, Strowd R, Skardal A. Exploration of Dynamic Elastic Modulus Changes on Glioblastoma Cell Populations With Aberrant EGFR Expression as a Potential Therapeutic Intervention Using a Tunable Hyaluronic Acid Hydrogel Platform. Gels (2017) 3:479-83. doi: 10.3390/gels3030028

145. Lian S, Shi R, Huang X, Hu X, Song S, Bai Y, et al. Artesunate Attenuates Glioma Proliferation, Migration and Invasion by Affecting Cellular Mechanical Properties. Oncol Rep (2016) 36:984-90. doi: 10.3892/ or.2016.4847

146. Brusatin G, Panciera T, Gandin A, Citron A, Piccolo S. Biomaterials and Engineered Microenvironments to Control YAP/TAZ-Dependent Cell Behaviour. Nat Mater (2018) 17:1063-75. doi: 10.1038/s41563-018-0180-8
147. Wolf KJ, Chen J, Coombes J, Aghi MK, Kumar S. Dissecting and Rebuilding the Glioblastoma Microenvironment With Engineered Materials. Nat Rev Mater (2019) 4:651-68. doi: 10.1038/s41578-019-0135-y

148. Baruffaldi D, Palmara G, Pirri C, Frascella F. 3d Cell Culture: Recent Development in Materials With Tunable Stiffness. ACS Appl Bio Materials (2021) 4:2233-50. doi: 10.1021/acsabm.0c01472

149. Pedron S, Harley BA. Impact of the Biophysical Features of a 3D Gelatin Microenvironment on Glioblastoma Malignancy. J BioMed Mater Res A (2013) 101:3404-15. doi: 10.1002/jbm.a.34637

150. Chauhan VP, Martin JD, Liu H, Lacorre DA, Jain SR, Kozin SV, et al. Angiotensin Inhibition Enhances Drug Delivery and Potentiates Chemotherapy by Decompressing Tumour Blood Vessels. Nat Commun (2013) 4:2516. doi: 10.1038/ncomms3516

151. Munson JM, Shieh AC. Interstitial Fluid Flow in Cancer: Implications for Disease Progression and Treatment. Cancer Manag Res (2014) 6:317-28. doi: 10.2147/CMAR.S65444

152. Sengul E, Elitas M. Single-Cell Mechanophenotyping in Microfluidics to Evaluate Behavior of U87 Glioma Cells. Micromachines (Basel) (2020) 11:111. doi: $10.3390 / \mathrm{mil} 1090845$

153. Jamin Y, Boult JKR, Li J, Popov S, Garteiser P, Ulloa JL, et al. Exploring the Biomechanical Properties of Brain Malignancies and Their Pathologic Determinants In Vivo With Magnetic Resonance Elastography. Cancer Res (2015) 75:1216-24. doi: 10.1158/0008-5472.CAN-14-1997

154. Tanner K, Boudreau A, Bissell MJ, Kumar S. Dissecting Regional Variations in Stress Fiber Mechanics in Living Cells With Laser Nanosurgery. Biophys J (2010) 99:2775-83. doi: 10.1016/j.bpj.2010.08.071

155. Budday S, Ovaert TC, Holzapfel GA, Steinmann P, Kuhl E. Fifty Shades of Brain: A Review on the Mechanical Testing and Modeling of Brain Tissue. Arch Comput Methods Eng (2020) 27:1187-230. doi: 10.1007/s11831-01909352-w

156. Yecies D, Liba O, Zerda A, Grant GA. Intraoperative Imaging Modalities and the Potential Role of Speckle Modulating Optical Coherence Tomography. Neurosurgery (2018) 65:74-7. doi: 10.1093/neuros/nyy199

157. Logun M, Zhao W, Mao L, Karumbaiah L. Microfluidics in Malignant Glioma Research and Precision Medicine. Adv Biosyst (2018) 2:1187-230. doi: 10.1002/adbi.201700221

158. Garcia CA, Bhargav AG, Brooks M, Suárez-Meade P, Mondal SK, Zarco N, et al. Functional Characterization of Brain Tumor-Initiating Cells and Establishment of GBM Preclinical Models That Incorporate Heterogeneity, Therapy, and Sex Differences. Mol Cancer Ther (2021) 65:74-7. doi: 10.1158/ 1535-7163.MCT-20-0547

159. Lee J, Kay K, Troike K, Ahluwalia MS, Lathia JD. Sex Differences in Glioblastoma Immunotherapy Response. Neuromolecular Med (2021) 2:143. doi: 10.1007/s12017-021-08659-x

160. Sun T, Plutynski A, Ward S, Rubin JB. An Integrative View on Sex Differences in Brain Tumors. Cell Mol Life Sci (2015) 72:3323-42. doi: 10.1007/s00018-015-1930-2

161. Lathia JD. Fountain of Chaos: Cerebrospinal Fluid Enhancement of Cancer Stem Cells in Glioblastoma. Neuro Oncol (2021) 23:530-2. doi: 10.1093/ neuonc/noab026

162. Lara-Velazquez M, Zarco N, Carrano A, Phillipps J, S Norton E, Schiapparelli P, et al. Alpha 1-Antichymotrypsin Contributes to Stem Cell Characteristics and Enhances Tumorigenicity of Glioblastoma. Neuro Oncol (2021) 23:599-610. doi: 10.1093/neuonc/noaa264

163. Rao SS, Bentil S, De Jesus J, Larison J, Hissong A, Dupaix R, et al. Inherent Interfacial Mechanical Gradients in 3D Hydrogels Influence Tumor Cell Behaviors. PloS One (2012) 7:e35852. doi: 10.1371/journal.pone.0035852

164. Pedron S, Becka E, Harley BA. Spatially Gradated Hydrogel Platform as a 3D Engineered Tumor Microenvironment. Adv Mater (2015) 27:1567-72. doi: 10.1002/adma.201404896

165. Schneider CS, Bhargav AG, Perez JG, Wadajkar AS, Winkles JA, Woodworth GF, et al. Surface Plasmon Resonance as a High Throughput Method to Evaluate Specific and non-Specific Binding of Nanotherapeutics. J Control Release (2015) 219:331-44. doi: 10.1016/j.jconrel.2015.09.048

166. Pedron S, Becka E, Harley BA. Regulation of Glioma Cell Phenotype in 3D Matrices by Hyaluronic Acid. Biomaterials (2013) 34:7408-17. doi: 10.1016/ j.biomaterials.2013.06.024 
167. Momin A, Bahrampour S, Min H-K, Chen X, Wang X, Sun Y, et al. Channeling Force in the Brain: Mechanosensitive Ion Channels Choreograph Mechanics and Malignancies. Trends Pharmacol Sci (2021) 42:367-84. doi: 10.1016/j.tips.2021.02.006

Conflict of Interest: The authors declare that the research was conducted in the absence of any commercial or financial relationships that could be construed as a potential conflict of interest.

Publisher's Note: All claims expressed in this article are solely those of the authors and do not necessarily represent those of their affiliated organizations, or those of the publisher, the editors and the reviewers. Any product that may be evaluated in this article, or claim that may be made by its manufacturer, is not guaranteed or endorsed by the publisher.

Copyright $\odot 2022$ Bhargav, Domino, Chamoun and Thomas. This is an open-access article distributed under the terms of the Creative Commons Attribution License (CC BY). The use, distribution or reproduction in other forums is permitted, provided the original author(s) and the copyright owner(s) are credited and that the original publication in this journal is cited, in accordance with accepted academic practice. No use, distribution or reproduction is permitted which does not comply with these terms. 